\title{
microRNA-21-5p dysregulation in exosomes derived from heart failure patients impairs regenerative potential
}

\author{
Li Qiao, ${ }^{1,2,3}$ Shiqi Hu, ${ }^{2,3}$ Suyun Liu, ${ }^{1}$ Hui Zhang, ${ }^{1}$ Hong Ma, ${ }^{4}$ Ke Huang, ${ }^{2,3}$ Zhenhua Li, ${ }^{2,3}$ Teng Su, ${ }^{2,3}$ Adam Vandergriff, ${ }^{2,3}$ \\ Junnan Tang, ${ }^{2,3,5}$ Tyler Allen, ${ }^{2,3}$ Phuong-Uyen Dinh, ${ }^{2,3}$ Jhon Cores, ${ }^{2,3}$ Qi Yin, ${ }^{2,3}$ Yongjun Li, ${ }^{1}$ and Ke Cheng ${ }^{2,3}$ \\ 'Department of Cardiology, The Second Hospital of Hebei Medical University, Shijiazhuang, China. 'Department of Molecular Biomedical Science, North Carolina State University, Raleigh, \\ North Carolina, USA. ${ }^{3}$ Department of Biomedical Engineering, University of North Carolina, Chapel Hill and North Carolina State University, Raleigh, North Carolina, USA. ${ }^{4}$ Department of Pathology and \\ Laboratory Medicine, University of North Carolina, Chapel Hill, North Carolina, USA. 5Department of Cardiology, The First Affiliated Hospital of Zhengzhou University, Zhengzhou, China.
}

Exosomes, as functional paracrine units of therapeutic cells, can partially reproduce the reparative properties of their parental cells. The constitution of exosomes, as well as their biological activity, largely depends on the cells that secrete them. We isolated exosomes from explant-derived cardiac stromal cells from patients with heart failure (FEXO) or from normal donor hearts (NEXO) and compared their regenerative activities in vitro and in vivo. Patients in the FEXO group exhibited an impaired ability to promote endothelial tube formation and cardiomyocyte proliferation in vitro. Intramyocardial injection of NEXO resulted in structural and functional improvements in a murine model of acute myocardial infarction. In contrast, FEXO therapy exacerbated cardiac function and left ventricular remodeling. microRNA array and PCR analysis revealed dysregulation of miR-21-5p in FEXO. Restoring miR-21-5p expression rescued FEXO's reparative function, whereas blunting miR-21-5p expression in NEXO diminished its therapeutic benefits. Further mechanistic studies revealed that miR-21-5p augmented Akt kinase activity through the inhibition of phosphatase and tensin homolog. Taken together, the heart failure pathological condition altered the miR cargos of cardiac-derived exosomes and impaired their regenerative activities. miR-21-5p contributes to exosome-mediated heart repair by enhancing angiogenesis and cardiomyocyte survival through the phosphatase and tensin homolog/Akt pathway.

\section{Introduction}

Mounting lines of evidence, including that from our laboratory (1-4), have demonstrated that cell-based therapy holds great promise for the regeneration of injured heart muscle (5-12). Cellbased products must be carefully preserved to maintain their viability and activity until transplantation, however, and there are also some risks involved in cell transplantation. The modes of action for cell therapy products remain elusive, making it difficult to standardize each cell lot. Recent meta-analyses indicate that cardiac cell therapies are overwhelmingly safe and only show none-to-marginal efficacy $(13,14)$. The development of cell-free and nonliving therapeutics (e.g., proteins, RNAs) has the potential to revolutionize cardiovascular regenerative medicine. These therapeutics have compounded the evidence showing that the benefits of stem cell therapies mainly come from paracrine mechanisms, instead of the direct differentiation of injected stem cells into cardiomyocytes $(15,16)$. The injected cells secrete proteins and nucleic acids to promote endogenous repair $(17,18)$.

Exosomes are nanosized membrane vesicles secreted by most cell types, including stem cells and cancer cells $(19,20)$. They are

Authorship note: LQ, SH, and SL contributed equally to this work. Conflict of interest: KC is an equity holder of Xollent Biotech Inc. Copyright: () 2019, American Society for Clinical Investigation. Submitted: June 25, 2018; Accepted: March 12, 2019; Published: April 29, 2019 Reference information: J Clin Invest. 2019;129(6):2237-2250. https://doi.org/10.1172/JCl123135. extracellular nanoshuttles that facilitate cell-cell communications and are crucial for maintaining cells' normal physiological functions. It has been reported that exosomes, as functional paracrine units of stem cells, can partially recapitulate the regenerative activities of their parent cells, suggesting that they may provide an alternative, cell-free therapeutic option (21-24). The mechanisms that drive the exosome-mediated repair processes rely largely on the transferring of exosomal cargos, including microRNAs (miR), mRNA, and proteins, to the recipient cells (25). Accordingly, the constitution of exosomes, as well as their biological activity largely depend on the physiological state of their parent cells (26-29).

It has been reported that stem cells derived from animals or patients with physiological stresses, such as systemic inflammation, have impaired regenerative activity (30-33). Because many patients with cardiac diseases, especially heart failure, suffer from these physiological stresses, we have hypothesized that cardiac cell-secreted exosomes derived from these patients may have impaired therapeutic activity, or possibly make the condition worse. In this article, we compared the treatment effects of cardiac exosomes from healthy donor hearts (normal exosomes, or NEXO) to those of cardiac exosomes from failing hearts (failure exosomes, or FEXO).

\section{Results}

Generation and characterization of NEXO and FEXO. Information regarding patients and healthy donors is presented in Table 1. There were no substantial differences in age or body mass index 


\section{Table 1. Patient and healthy heart donor characteristics}

\begin{tabular}{|c|c|c|c|}
\hline \multicolumn{4}{|l|}{ Normal human } \\
\hline ID & №. 1 & NNo. 2 & No. 3 \\
\hline Sex & Male & Male & Male \\
\hline Age (yr) & 43 & 18 & 41 \\
\hline Race & Hispanic & Hispanic & Caucasian \\
\hline Cause of death & $\begin{array}{c}\text { Head trauma/motor } \\
\text { vehicle accident }\end{array}$ & $\begin{array}{c}\text { Head trauma/motor } \\
\text { vehicle accident }\end{array}$ & $\begin{array}{c}\text { Head trauma/motor } \\
\text { vehicle accident }\end{array}$ \\
\hline \multicolumn{4}{|l|}{ Patients with heart failure } \\
\hline ID & №. 1 & №. 2 & No. 3 \\
\hline Age (yr) & 44 & 54 & 60 \\
\hline Sex & Female & Female & Male \\
\hline Weight (kg) & 78 & 62 & 116 \\
\hline Body mass index & 32.5 & 26.6 & 35.6 \\
\hline Smoker & No & No & Yes \\
\hline Diabetes & No & Yes & No \\
\hline Hyperlipidemia & Yes & Yes & Yes \\
\hline Renal dysfunction & No & No & No \\
\hline Hypertension & Yes & Yes & Yes \\
\hline Chronic lung disease & No & No & Yes \\
\hline Peripheral vascular disease & No & No & No \\
\hline Cerebrovascular disease & Yes & No & No \\
\hline Myocardial infarction & No & No & No \\
\hline Coronary artery disease & No & No & Yes \\
\hline Angina & Yes & No & No \\
\hline Arrhythmia & Yes & No & Yes \\
\hline Congestive heart failure & Yes & Yes & Yes \\
\hline Classification NYHA ${ }^{A}(I / I I / I I I / I V)$ & IV & IV & IV \\
\hline Cardiac surgical procedure & No & No & No \\
\hline
\end{tabular}

between the 2 groups. Explant-derived cardiac stromal cells were harvested from the donors' hearts (see Cell Culture and Exosome Isolation in Methods, Supplemental Figure 1A and B; supplemental material available online with this article; https://doi.org/10.1172/ JCI123135DS1). Flow cytometry revealed that explant-derived cells contain a negligible subpopulation of $\mathrm{c}-\mathrm{kit}^{+}$cells and endothelial cells $\left(\mathrm{CD} 31^{+}, \mathrm{CD} 34^{+}\right)$, along with a dominating subpopulation that phenotypically resembles mesenchymal cells or fibroblasts $\left(\mathrm{CD}^{+}, \mathrm{CD} \mathrm{C5}^{+}\right)$(Supplemental Figure 2). NEXO and FEXO were isolated from the 14-day conditioned media of cardiac cells from healthy heart donors or patients with heart failure, respectively (Supplemental Figure 1A). Cell viability was confirmed at the end of the conditioning period (Supplemental Figure 1, B and C). Cardiac cells from patients with heart failure and those from healthy heart donors had comparable exosome yields (Supplemental Figure 3A) and exosome sizes (Supplemental Figure 3B), as measured by NanoSight nanoparticle tracking analysis. Transmission electron microscopy revealed the vesicular morphology of exosomes and confirmed their size did not exceed $200 \mathrm{~nm}$ (ref. 34 and Supplemental Figure 3D). In addition, Western blot analysis indicated the presence of signature exosomal markers, such as protein CD81, Alix, and TSG101 (Supplemental Figure 3C). Both types of exosomes were abundant in RNA, and the amount of RNA contents in NEXO and FEXO was indistinguishable (Supplemental Figure 3E).
Effects of NEXO or FEXO on cardiomyocytes, endothelial cells, and cardiac fibroblasts in vitro. Cardiomyocytes, endothelial cells, and cardiac fibroblasts (CFs) are the 3 major cell types in the heart. We first measured the uptake efficiency of NEXO and FEXO by those cells. DiI-labeled exosomes were readily internalized by neonatal rat cardiomyocytes (NRCMs) (Figure 1A). There was no significant difference in uptake efficiency between NEXO and FEXO by NRCMs (Figure 1B). NEXO significantly promoted cardiomyocyte proliferation, as evidenced by the higher percentage of Ki-67-positive nuclei, while treatment with FEXO suppressed cardiomyocyte proliferation (Figure 1, C and D). In addition, cardiomyocytes treated with NEXO exhibited fewer terminal deoxynucleotidyl transferase nick-end labeling (TUNEL)-positive nuclei, indicating less apoptosis. In contrast, FEXO did not show any antiapoptotic effects (Figure 1, E and F). To further validate the effects of NEXO and FEXO on adult cardiomyocytes, human cardiomyocytes (HCs) were cultured on HC cell culture extracellular matrix, then treated with NEXO or FEXO for 24 hours. NEXO significantly promoted cardiomyocyte proliferation, as indicated by the higher percentage of Ki-67, while treatment with FEXO suppressed cardiomyocyte proliferation (Supplemental Figure 4, A and D). This result was supported by the assessment of 2 additional proliferation markers: phosphohistone $\mathrm{H} 3$, a characteristic marker of mitosis, and aurora kinase B, a cytokinesis marker, (Supplemental Figure 4, B-F). Furthermore, adult cardiomyocytes treated with NEXO exhibited fewer TUNEL-positive nuclei, suggesting less apoptosis. In contrast, treatment with FEXO did not show any antiapoptotic effects (Supplemental Figure 4, G and H).

A tube formation assay using human umbilical vein endothelial cells (HUVECs) was used to evaluate the proangiogenic effects of exosomes. Treatment with FEXO inhibited tube formation of HUVECs, while NEXO promoted tube formation, compared to saline control (Figures $1, \mathrm{G}$ and $\mathrm{H}$ ). A-Smooth muscle actin ( $\alpha$-SMA) was employed as an indicator of transition from fibroblasts to myofibroblasts. The expression of $\alpha$-SMA was upregulated in FEXO-treated CFs (Figures 1, I and J), suggesting a phenotype transition to myofibroblasts. Taken together, our data suggest that cardiac cells in the failing heart secreted exosomes with an impaired ability to promote cardiomyocyte proliferation, decrease programmed cell death, and stimulate angiogenesis in vitro. Moreover, such diseased exosomes drive the differentiation of fibroblasts to myofibroblasts.

Effects of NEXO and FEXO therapy on cardiac function and morphometry in a mouse model of acute myocardial infarction. We induced an acute myocardial infarction (MI) in CD1 mice by coronary vessel ligation, then intramyocardially injected NEXO, FEXO, or phosphate-buffered saline (PBS) into the MI border zone. Left ventricular ejection fraction (LVEF) was used to assess 
A

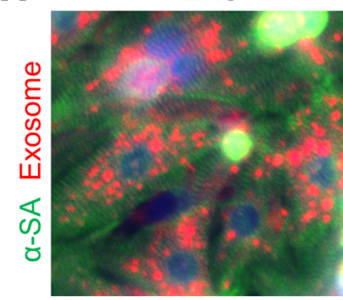

C

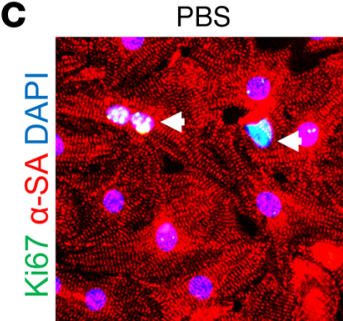

E

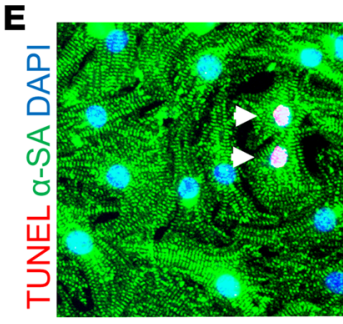

\section{G}

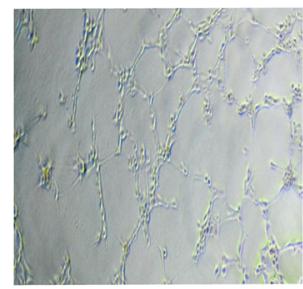

I

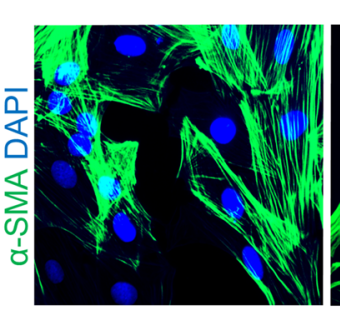

FEXO

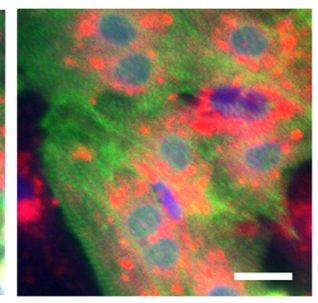

FEXO
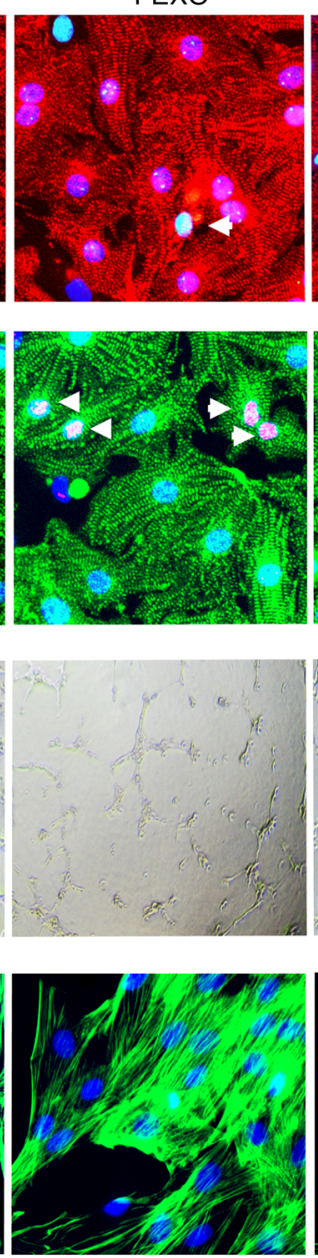

B
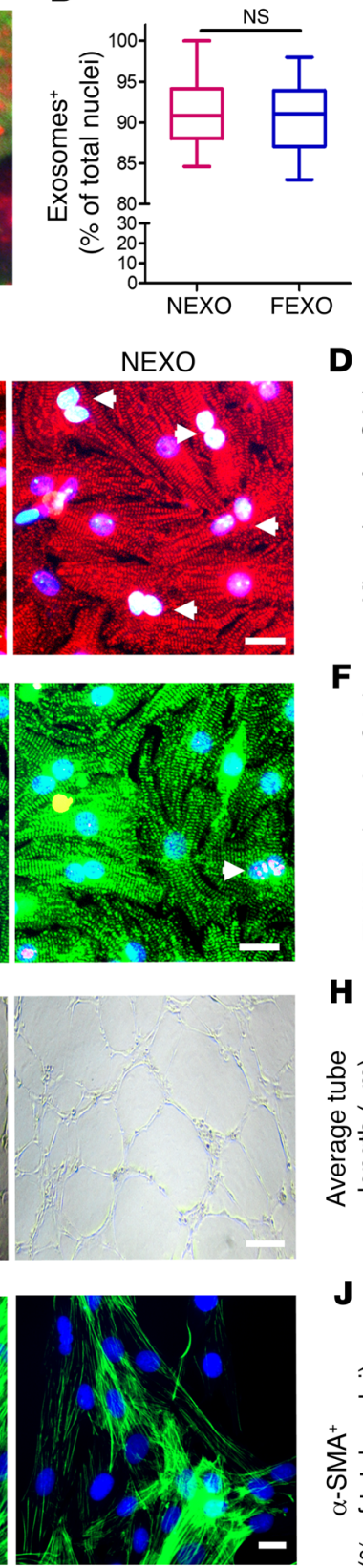

\section{D}

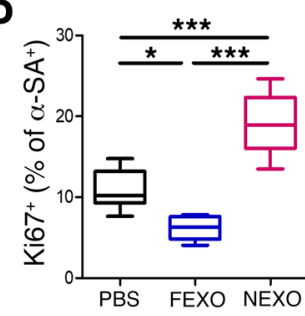

$\mathbf{F}$

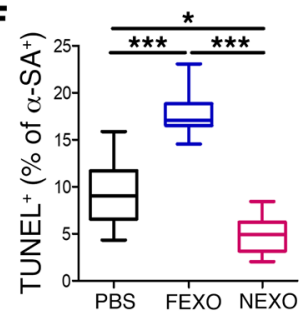

H

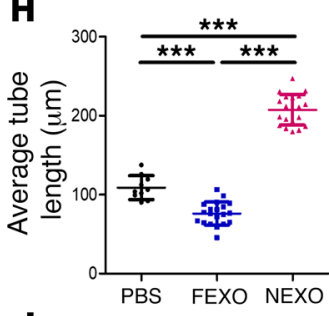

J

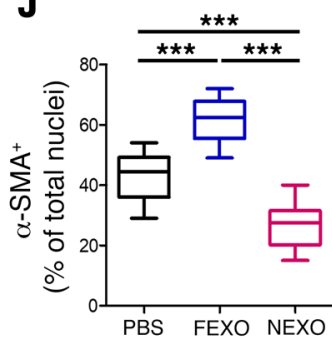

Figure 1. Effects of exosome treatment on cardiomyocytes, endothelial cells, and cardiac fibroblasts in vitro. (A) Representative fluorescent micrographs showing uptake of Dil-labeled NEXO and FEXO by NRCMs. Endocytosed exosomes (red) can be seen within the cytoplasm of cardiomyocytes (green). Scale bar: $10 \mu \mathrm{m}$. (B) Quantitation of exosomes uptake $(n=10)$. Twotailed $t$ test. (C) NRCM proliferation in response to NEXO, FEXO, or PBS treatment. White arrows indicate Ki- $67^{+} / \alpha-\mathrm{SA}^{+}$cells. Scale bar: $20 \mu \mathrm{m}$. (D) Quantitation of proliferating cardiomyocytes $(n=6)$. (E) Apoptotic NRCMs in response to NEXO, FEXO, or PBS treatment. White arrows indicate $\mathrm{TUNEL}^{+} / \alpha-\mathrm{SA}^{+}$cells. Scale bar: $20 \mu \mathrm{m}$. (F) Quantitation of apoptotic cardiomyocytes $(n=6)$. (C) Measurement of tube formation in HUVECs co-cultured with NEXO, FEXO, or PBS. Scale bar: $100 \mu \mathrm{m}$. (H) Quantitation of average HUVEC tube length $(n=20)$. (I) Neonatal rat fibroblasts underwent phenotypic transition to myofibroblasts in response to NEXO, FEXO, or PBS treatment. Scale bar: $20 \mu \mathrm{m}$. (J) Quantitation of myofibroblasts $(n=12) .{ }^{*} P<0.05,{ }^{* * *} P<0.001$. NS, no significance. (D, F, $\mathbf{H}$, and J) One-way ANOVA with Bonferroni post hoc correction. All values are mean $\pm \mathrm{SD}$. FEXO, exosomes derived from the cardiac cells of patients with heart failure. NEXO, exosomes derived from the cardiac cells of normal heart donors. the enhancement or preservation of heart function. There was no significant difference in LVEF between the 3 treatment groups at baseline (Figure 2A). LVEF progressively decreased in the control group over the next 3 weeks, whereas hearts injected with NEXO showed greater LVEFs, compared to those treated with FEXO or PBS control. FEXO-treated hearts exhibited a worsening of LVEFs when compared to PBS-treated control hearts (Figure 2B). To facilitate head-to-head comparisons, treatment effect was evaluated by calculating the changes in endpoint LVEF relative to baseline LVEF. Control treatment had a negative effect, as LVEF declined over time, while NEXO treatment robustly prevented the decline of LVEF post-MI (Figure 2C). Likewise, the animals that received NEXO treatment showed a recovery in fractional shortening, whereas those injected with FEXO suffered from fractional shortening deterioration (Supplemental Figure 5A). These results demonstrate that FEXO has reduced reparative activity and may exacerbate LV dysfunction post-MI. At the histological level, Masson's trichrome staining was performed to simultaneously explore infarcted (blue) and viable (red) cardiac tissues (Figure 2D). NEXO-treated hearts exhibited decreased infarct size (Figure 2E), increased infarcted wall thickness (Figure $2 \mathrm{~F}$ ), and increased viable tissue (Figure 2G) compared to FEXO and PBS controls. Similarly, injured hearts treated with NEXO displayed less chamber dilation (Supplemental Figure 5B and C) and smaller infarct circumference (Supplemental Figure 5D) when compared to control or FEXO-treated hearts. These data indicated that NEXO attenuated LV remodeling after MI, whereas FEXO exacerbates it. 
A

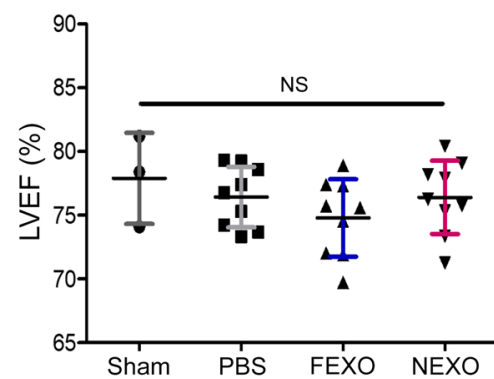

B

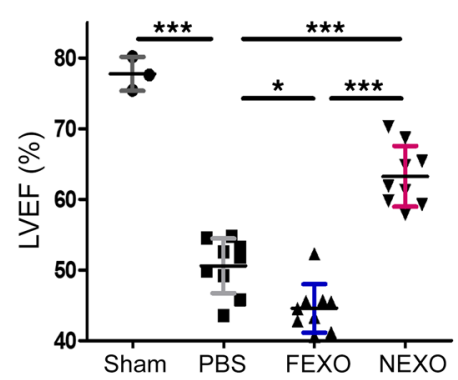

C Treatment effects

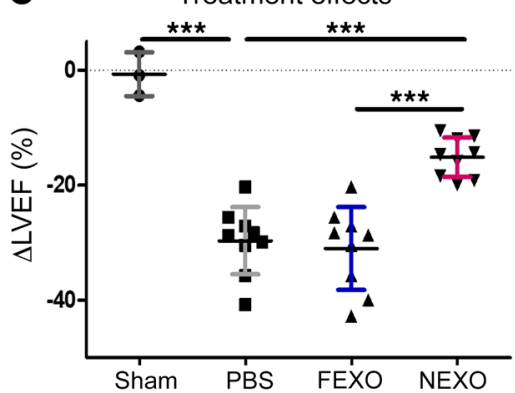

D
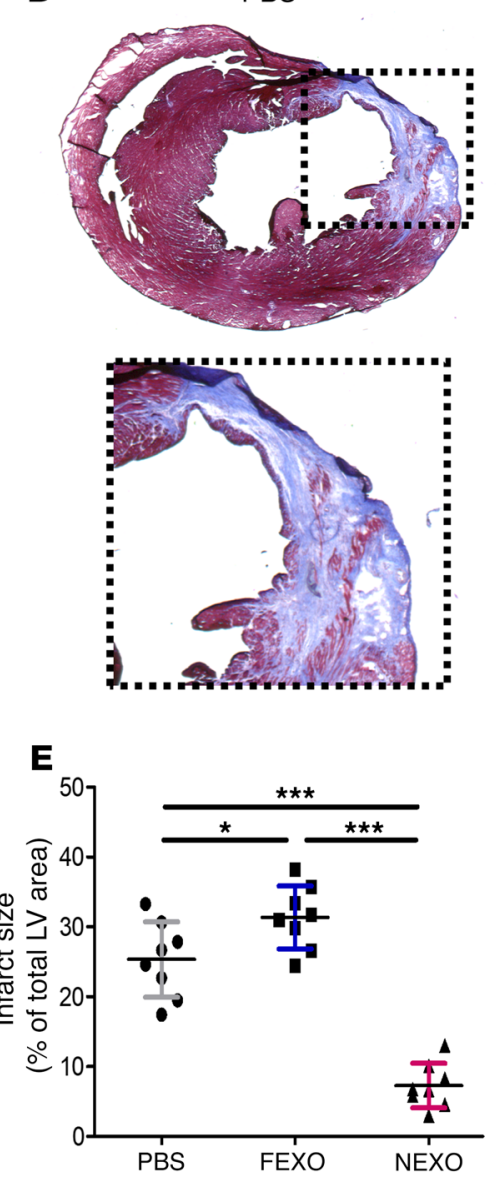
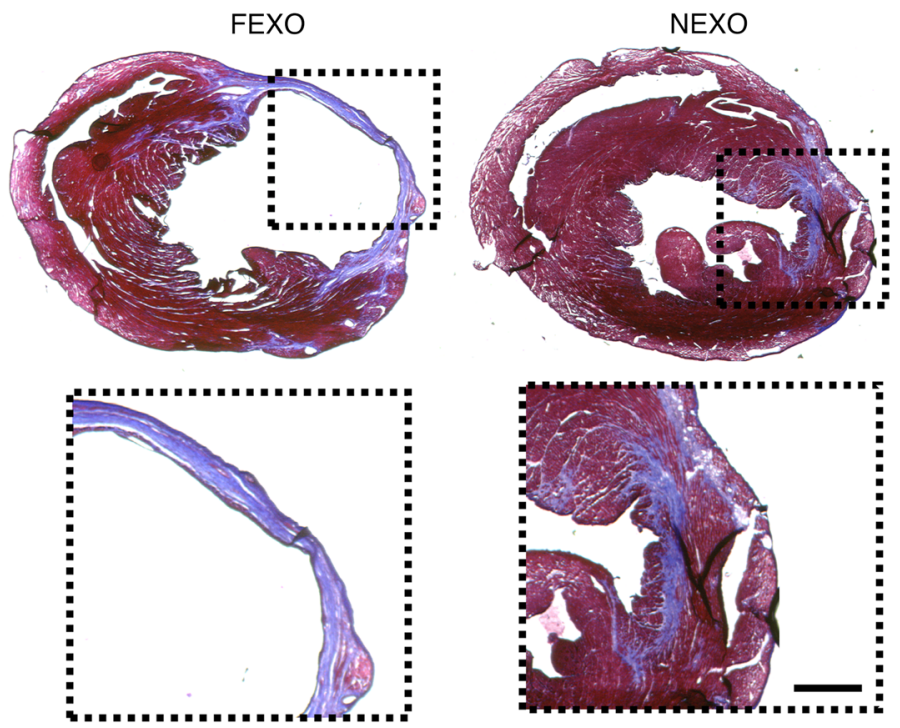

$\mathbf{F}$

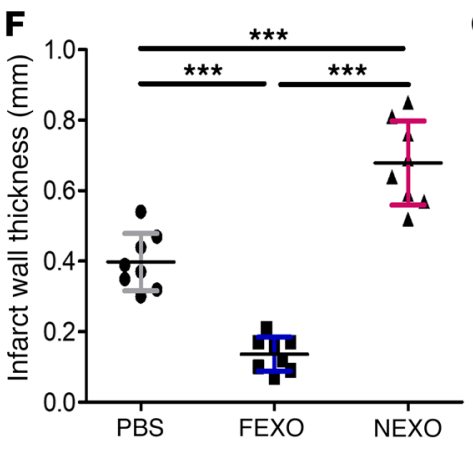

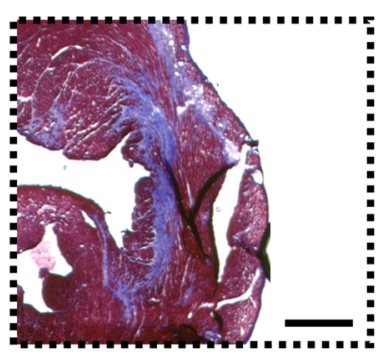

G

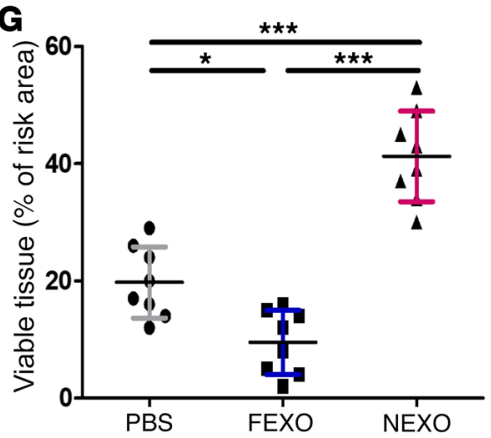

Figure 2. Effects of NEXO and FEXO treatment in a mouse model of acute MI. (A and B) LVEF was measured by echocardiography at baseline (1 day before MI) (A) and endpoint ( 3 weeks after MI) (B). (C) Treatment effects (changes in LVEF at 3 weeks relative to baseline) in each group (A-C, $n=9$ animals per treatment group, $n=3$ animals for the sham group). (D) Representative Masson's trichrome staining of myocardial section 3 weeks after treatment with NEXO, FEXO, or PBS. Scale bar: $0.5 \mathrm{~mm}$. (E-C) Quantitative analyses of infarct size, infarct wall thickness, and viable tissue from Masson's trichrome-stained heart sections ( $n=8$ animals per treatment group). ${ }^{*} P<0.05$, ${ }^{* *} P<0.001$. One-way ANOVA with Bonferroni post hoc correction. All values are mean \pm SD. FEXO, exosomes derived from the cardiac cells of patients with heart failure. NEXO, exosomes derived from the cardiac cells of normal heart donors.

Effects of NEXO and FEXO treatment on post-MI heart angiomyogenesis and apoptosis. It has been well established that adult stem cells exert their therapeutic benefits though indirect regenerative mechanisms. The secretome from the injected cells enhances cardiomyocyte proliferation, promotes neovascularization, and inhibits cardiomyocyte apoptosis $(15,35)$. Three weeks after MI and exosome injections, the percentage of cycling cardiomyocytes $\left(\mathrm{Ki}-67^{+} / \mathrm{a}-\mathrm{SA}^{+}\right.$, Figure $3, \mathrm{~A}$ and $\left.\mathrm{D}\right)$ in the peri-infarct zone of
NEXO-treated hearts was significantly higher than in those that received FEXO or PBS control injections. This finding was further confirmed by mitosis marker phospho-histone $\mathrm{H} 3$ and cytokinesis marker aurora kinase B (Supplemental Figure 6). A similar trend was detected in capillary density (von Willebrand [vWF] $]^{+}$capillaries, Figure 3, B and E), suggesting the proangiogenic role of NEXO but not of FEXO. Hearts injected with NEXO displayed significantly fewer apoptotic cardiomyocytes (TUNEL ${ }^{+} / \mathrm{a}-\mathrm{SA}^{+}$, Figure 

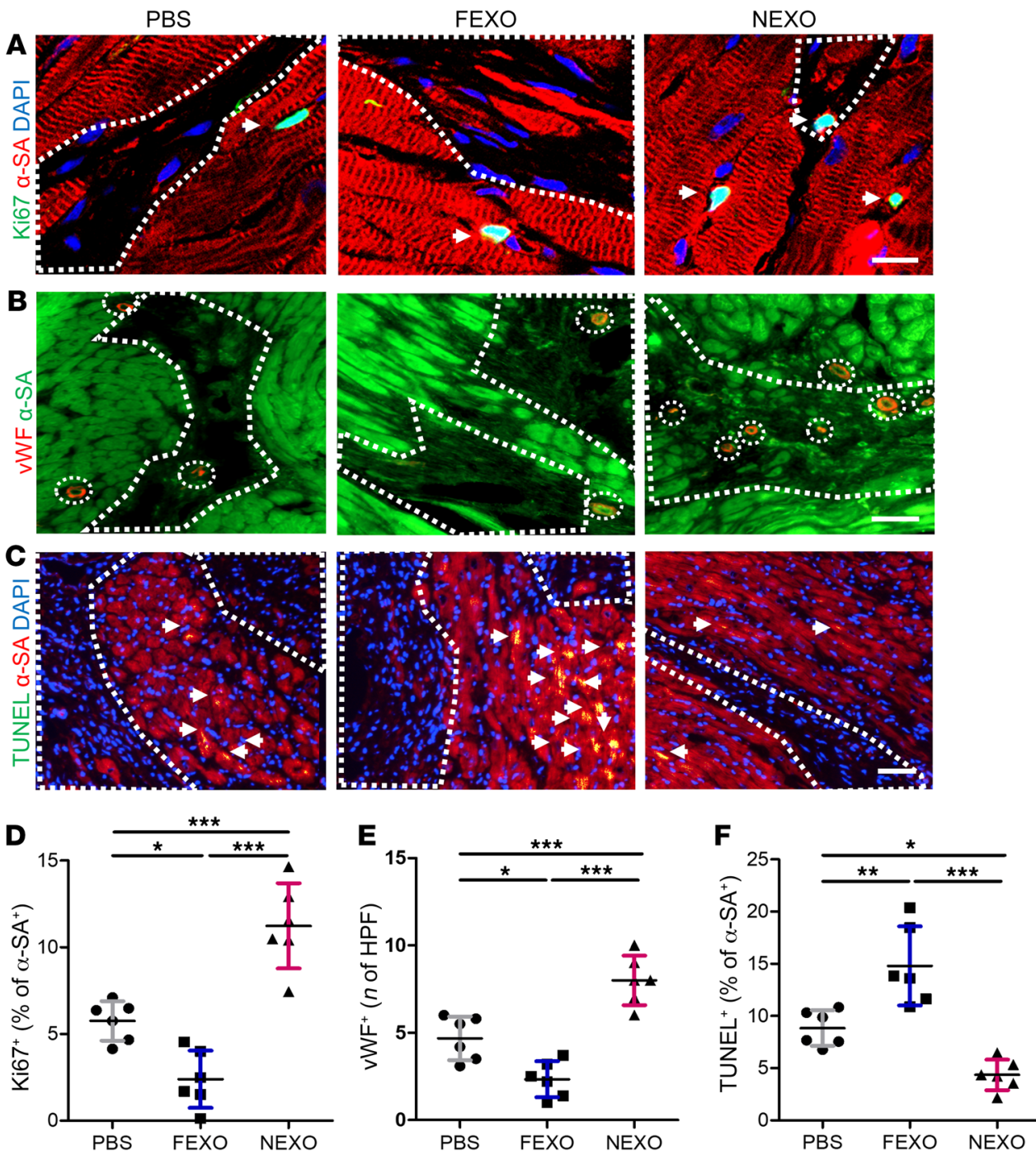

Figure 3. Mechanisms of exosomemediated cardiac repair. (A) Representative images of post-MI heart sections stained with $\mathrm{Ki}-67$ (green), $\alpha$-SA (red), and DAPI. White boundaries show infarct area, and white arrows indicate $\mathrm{Ki}-67^{+}$cells in the periinfarct zone. Scale bar: $10 \mu \mathrm{m}$ (B) Heart sections stained with VWF (red), $\alpha$-SA (green) in response to NEXO, FEXO, or PBS treatment. White arrows indicate capillary structures in the peri-infarct zone. Scale bar: $50 \mu \mathrm{m}$. (C) Heart sections stained with TUNEL (green), $\alpha-S A$ (red), and DAPI (blue). White arrows indicate apoptotic cardiomyocytes in the peri-infarct zone. Scale bar: $50 \mu \mathrm{m}$. (D) Quantification of cycling cardiomyocytes $\left(\mathrm{Ki}-67^{+} / \alpha-S A^{+}\right)$. (E) Quantification of capillary density (VWF+). (F) Quantification of cardiomyocyte apoptosis (TUNEL ${ }^{+} / \alpha-\mathrm{SA}^{+}$). (D-F) $n=6$ animals per group, 3 heart sections for each animal. ${ }^{*} P<0.05$, ${ }^{*} P<0.01,{ }^{* * *} P<0.001$. One-way ANOVA with Bonferroni post hoc correction. All values are mean $\pm \mathrm{SD}$. FEXO, exosomes derived from the cardiac cells of patients with heart failure. NEXO, exosomes derived from the cardiac cells of normal heart donors.
3, $\mathrm{C}$ and F), suggesting NEXO therapy led to tissue preservation. Overall, FEXO treatment is detrimental to post-MI healing, as it inhibits angiogenesis and cardiomyocyte proliferation.

Dysregulation of miR-21-5p in FEXO. Several studies imply that the mechanism of exosome-mediated repair involves exosomal miRNAs, which target specific signaling pathways in the recipient cells (e.g., injured cardiomyocytes) $(36,37)$. Therefore, we hypothesized that the functional impairment of FEXO might result from altered miR cargos. We compared the miRNA components of NEXO to that of FEXO using PCR microarrays for 84 wellreported miRNAs (Supplemental Figure 7, A and B). Twenty-three miRs were differentially expressed in the 2 groups of exosomes. Among those, miR-21-5p was the most dysregulated in FEXO, as compared to the expression in NEXO (Figure 4, A and B). We performed RT-PCR to verify the results. Interestingly, miR-21-5p, but not miR-21-3p, was dysregulated in FEXO (Figures 4C). We further verified that the myocardial tissue levels of miR-21-5p were decreased in the post-MI hearts injected with FEXO (Supplemental Figure 7C), as compared to those injected with NEXO.

The role of miR-21-5p in exosome-mediated heart repair. We designed various gain- and loss-of-function studies to verify the role of miR-21-5p in exosome-mediated, post-MI repair. The silencing of exosomal miR-21-5p by transfecting healthy cardiac cells with anti-miR-21-5p oligo (NEXO + anti-miR-21) was confirmed by RT-PCR on the exosome products (Supplemental Figure 8, A and C). Knocking down miR-21-5p in NEXO led to impaired antiapoptotic effects of NEXO on cultured H9C2 cells (Figure 5, A and B). In contrast to the control NEXO, NEXO + anti-miR-21 lost the ability to promote tube formation (Figure 5, C and D). In the meantime, we also engineered miR-21rescued exosomes by transfecting heart failure cardiac cells with miR-21 oligo. These cells were engineered to produce exosomes with miR-21-5p overexpression (FEXO + miR-21) (Supplemental Figure 8, A and B). As expected, upregulation of miR-21-5p in FEXO rescued their ability to inhibit apoptosis (Figure 5, E and F) and promote tube formation (Figure 5, G and H). To further validate the functions of miR-21 in adult cardiomyocytes, we transfected adult HCs with miR-21 mimic or scrambled control. Overexpression of miR-21 led to improved survival of adult cardiomyocytes, as indicated by less apoptotic cells (TUNEL-positive nuclei) (Supplemental Figure 9).

To confirm those results in vivo, we employed the same mouse MI model to test the therapeutic effects of FEXO + miR21 and NEXO + anti-miR-21. Mice injected with NEXO + anti- 

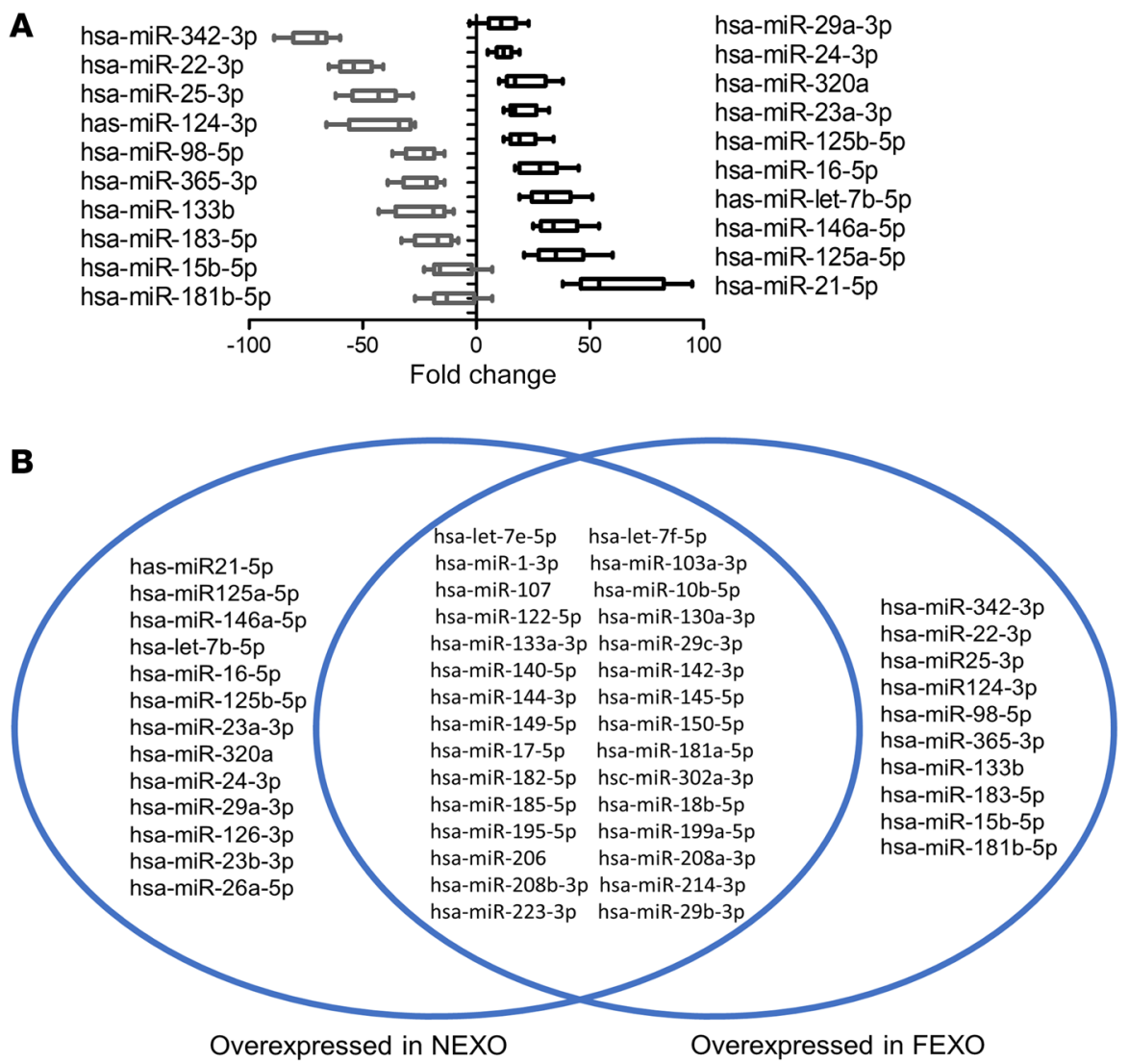

C

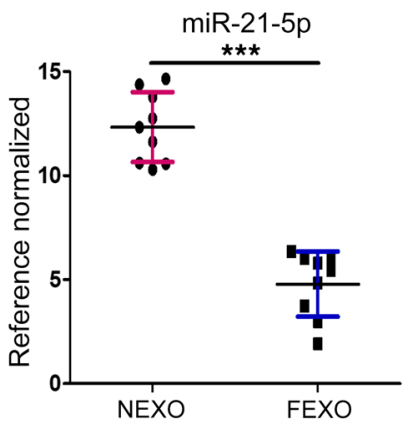

miR-21-3P

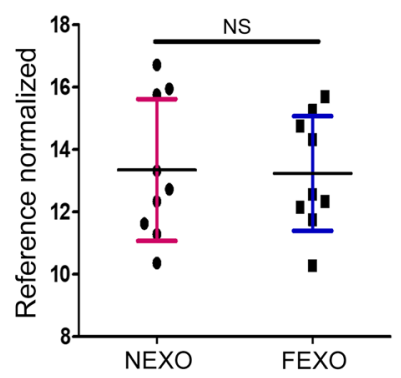

Figure 4. Dysregulation of miR-21-5p in heart failure exosomes. (A) miRNA array showing fold changes of miRNA abundance in NEXO or FEXO ( $n=3$ biological replicates, 3 technical replicates for each biological replicate). (B) Venn diagram showing the variable miRNA profile between NEXO and FEXO. (C) RT-PCR analysis validated that miR-21-5p was highly enriched in NEXO ( $n=3$ biological replicates, 3 technical replicates for each biological replicate).

${ }^{* * *} P<0.001$. NS, no significance. Two-tailed $t$ test. All values are mean $\pm \mathrm{SD}$. FEXO, exosomes derived from the cardiac cells of patients with heart failure. NEXO, exosomes derived from the cardiac cells of normal heart donors.
miR-21 exhibited decreased pump function (Figure 6, A and B), increased scar mass, and decreased viable heart tissue, compared to the hearts injected with $\mathrm{NEXO}+$ miR-scr (Figure 6, C-G). In contrast, miR-21-5p enhancement rescued the therapeutic functions of FEXO. FEXO + miR-21 treatment led to improvement in cardiac function (Figure 6, A and B) and attenuation of LV remodeling (Figure 6, D-G). Histological analysis revealed that miR-21$5 p$ inhibition abolished the ability of NEXO therapy to promote angiogenesis (Figure 7, A and B) and inhibit apoptosis (Figure 7, C and D). On the contrary, restoring miR-21-5p expression in FEXO rescued those paracrine effects (Figure 7, E-H). Moreover, the proangiogenic effects of miR-21-5p were confirmed by the increased number of functional vessels. The functionality of a blood vessel was validated by co-staining the endothelial cell marker, vWF, and the RBC marker. As shown in Supplemental Figure 10, RBCs were detected in the blood vessels in the MI border zone of the hearts treated with miR-21-overexpressed FEXO. Through those gain- and loss-of-function studies, we confirmed the pivotal role of miR-21-5p in exosome-mediated heart repair, possibly through proangiogenic and antiapoptotic mechanisms.

Together with miR-21, other miRs at the top of our list are let$7 b-5 p$, miR125a-5p, and miR-146a-5p. We explored the effects of those miRs on cardiomyocytes, CFs, and endothelial cells as well. The results indicated that miR-146a inhibited apoptosis in cardiomyocytes but promoted tube formation in endothelial cells (Supplemental Figure 11). This finding was consistent with previous studies (36). In contrast, neither let-7b-5p nor miR-125a-5p showed beneficial effects on cardiomyocyte survival or endothelial tube formation (Supplemental Figure 11).

Effects of miR-21-5p on phosphatase and tensin homolog/Akt signaling. It has been established that miR-21-5p promotes cancer cell proliferation, angiogenesis, migration, and invasion by targeting pathways involving phosphatase and tensin homolog (PTEN), programmed cell death 4 (PDCD4), Forkhead box O1, SMAD family member 7 , tumor necrosis factor- $\alpha$-induced protein-8-like 2, Sprouty2 (Spry2), etc. (38). Recent studies have 

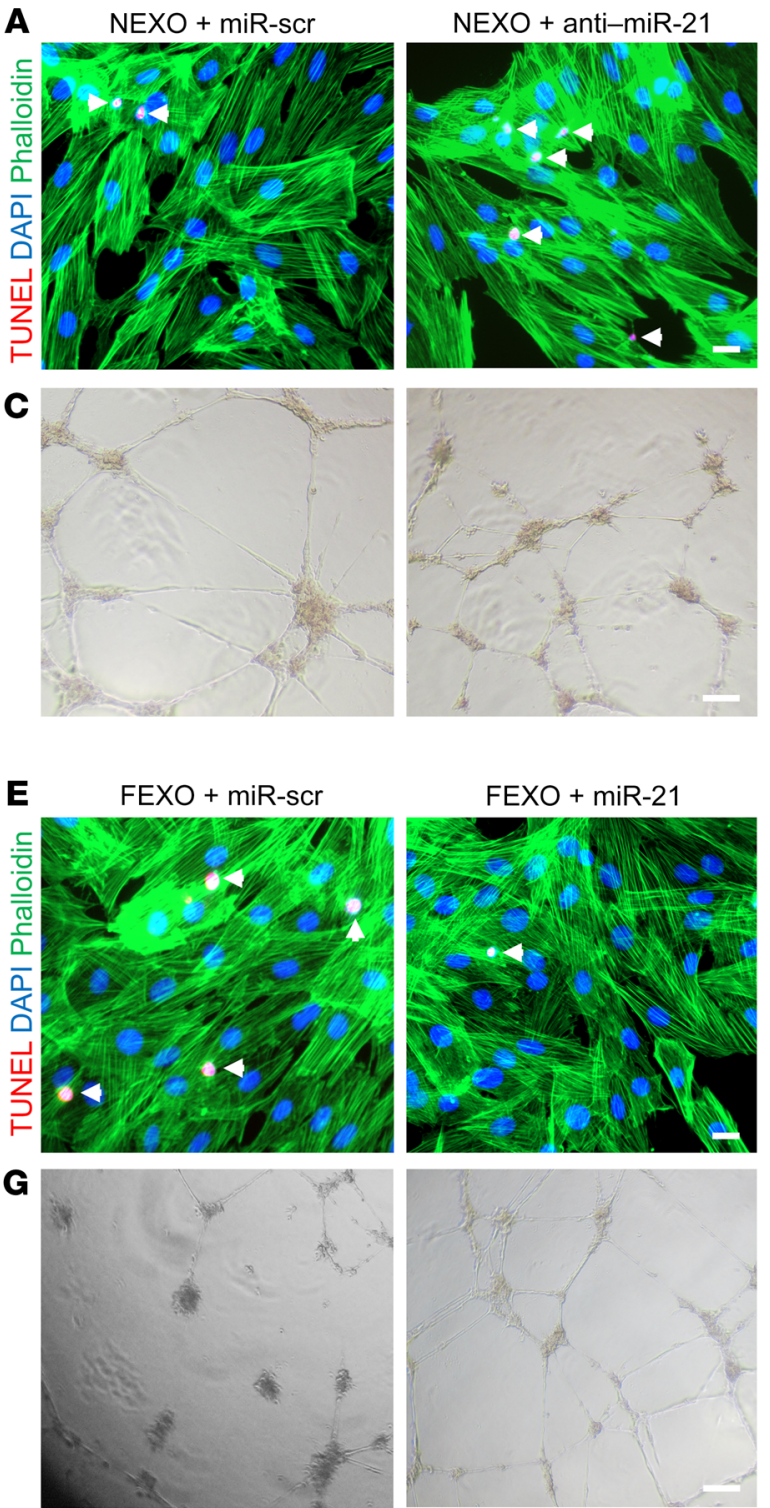

\section{$\mathbf{F}$}
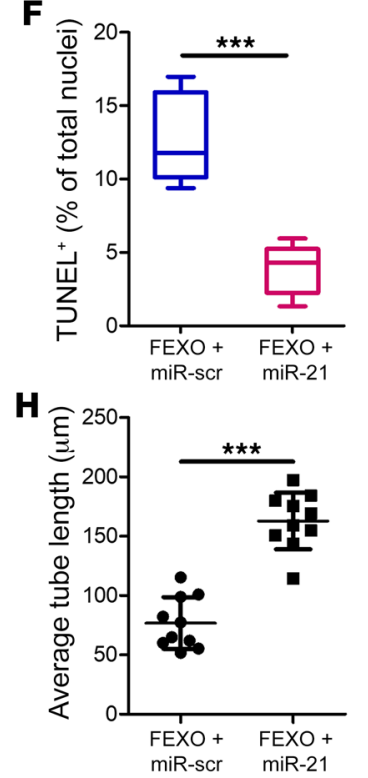

Figure 5. Manipulation of miR-21-5p in exosomes modulates their proangiogenic and antiapoptosis activities in vitro. (A) Antiapoptotic effects were diminished after miR-21-5p knockdown in NEXOs. White arrows indicate TUNEL $L^{+}$cells. Scale bar: $50 \mu \mathrm{m}$. (B) Quantitation of apoptotic cells ( $n$ =6). (C) The proangiogenic effects of NEXO were diminished by miR-21-5p knockdown. Scale bar: 50 $\mu \mathrm{m}$. (D) Quantitation of average tube length $(n=$ 10). (E) Enhancing miR-21-5p expression in FEXO partly rescued its ability to promote cardiomyocyte proliferation. Scale bar: $50 \mu \mathrm{m}$. (F) Quantitation of apoptotic cells $(n=6)$. (G) Tube formation assay showing enhanced proangiogenic activity of FEXO with restored miR-21-5p expression. Scale bar: 50 $\mu \mathrm{m}$. (H) Quantitation of average tube length $(n=$ 10). ${ }^{* *} P<0.001$. Two-tailed $t$ test. All values are mean $\pm \mathrm{SD}$. FEXO + miR-scr, exosomes derived from the cardiac cells of patients with heart failure transfected with scrambled miR oligo. FEXO + miR-21, exosomes derived from the cardiac cells of patients with heart failure transfected with miR-21-5p oligo. $\mathrm{NEXO}+$ miR-scr, exosomes derived from the cardiac cells of the normal hearts transfected with scrambled miR oligo. NEXO + anti-miR-21, exosomes derived from the cardiac cells of the normal hearts transfected with anti-miR-21-5p oligo. FEXO, exosomes derived from the cardiac cells of patients with heart failure. NEXO, exosomes derived from the cardiac cells of normal heart donors. also shown that PTEN and Spry1 are targeted by miR-21-5p in cardiovascular diseases $(39,40)$. To identify the potential target genes of miR-21 on 3 major cell types in the heart, we incubated cardiomyocytes (using human induced-pluripotent stem cell-derived cardiomyocytes [iCMs] and H9C2 cells), CFs (using human CFs), and endothelial cells (using HUVECs) with miR-21 mimic or scrambled control. We then examined 18 phosphorylated proteins with a signaling pathway phosphorylation array. The signaling pathway array pointed at PTEN, AKT, and BAD as the miR-21-regulated genes in cardiomyocytes and endothelial cells but not in CFs (Supplemental Figure 12).

Western blot studies further confirmed that PTEN expression was significantly attenuated in HCs and HUVECs treated with miR-21 mimics (Figure 8, A and B). This finding coincides with enhanced Akt phosphorylation, as well as the alteration of other downstream targets such as caspase-3 and Bcl-2 (Figure 8, A and $\mathrm{C}-\mathrm{E})$. Upregulation of miR-21 in cardiomyocytes and HUVECs also promoted proliferating cell nuclear antigen ([PCNA] a pro- liferation marker) and vascular endothelial growth factor (VEGF) expressions (Figure 8, F-H), which are consistent with the preceding data in Figure 5 showing the proangiogenic effects of miR-21. Previous studies indicated that PDCD4, a proapoptotic protein, is a known miR-21 target (41), so we checked PCDC4 expressions in CMs, HCFs, and HUVECs treated with miR-21 mimics. Western blot analysis showed reduced PDCD4 expression in CMs only, but not in HCFs or HUVECs (Figure 8, F and I).

Taken together, our data indicate that miR-21 inhibits cardiomyocyte apoptosis by targeting PDCD4, promotes angiogenesis by activating PTEN/Akt signaling, and promotes the expression of VEGF in endothelial cells. Interestingly, because the flow cytometry showed a significant proportion of mesenchymal cells or fibroblasts $\left(\mathrm{CD} \mathrm{O}^{+}, \mathrm{CD} 105^{+}\right)$in our cardiac cells (Supplemental Figure 2), it is highly possible that those cardiac cells secreted exosomes containing miR-21 to communicate with nearby cardiomyocytes and endothelial cells under physiological and heart failure conditions. 

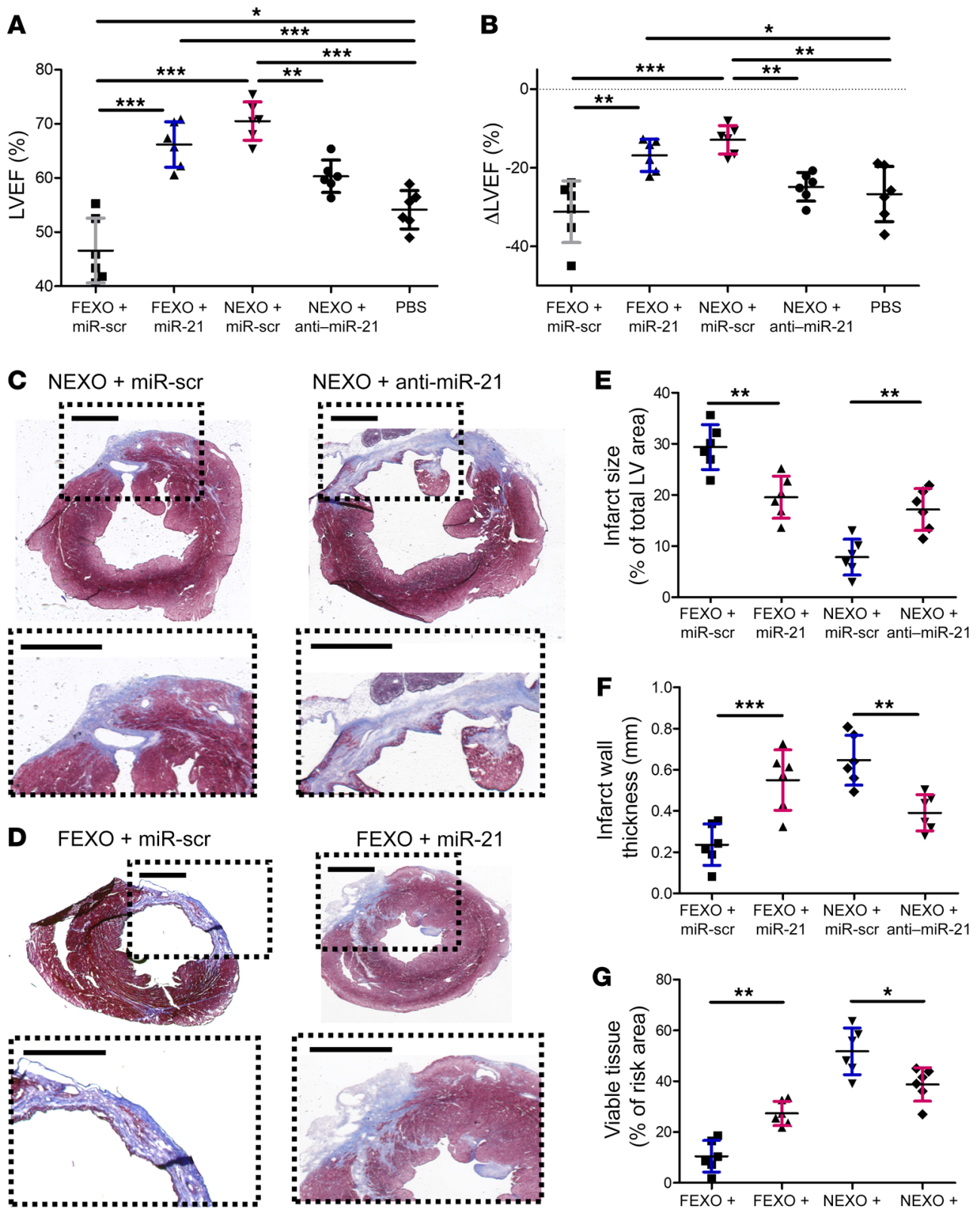
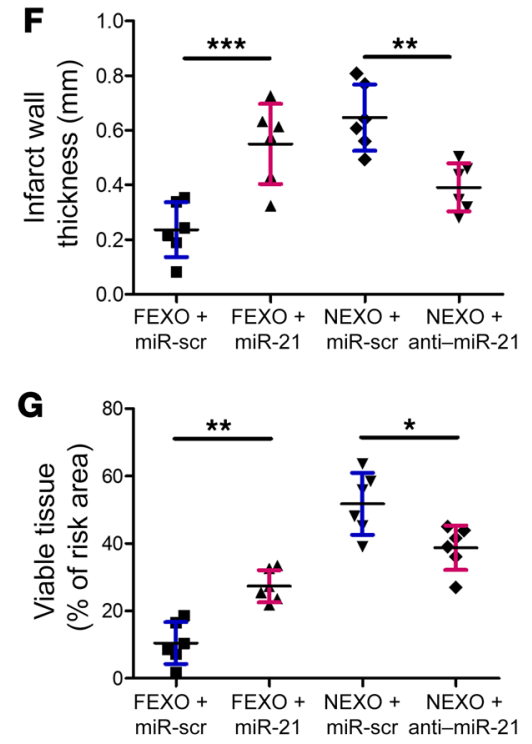

Figure 6. Manipulation of miR21 in exosomes modulates their therapeutic potency. miR-21-5p-deficient exosomes were produced by transfecting healthy cardiac cells with anti-miR21-5p oligo (NEXO + anti-miR-21). miR-21-5p-restored exosomes were engineered by transfecting heart failure cardiac cells with miR-21-5p oligo (FEXO + miR-21), followed by media conditioning and exosome isolation, as previously described. Scrambled miR oligo was used as a control (NEXO/FEXO + miR-scr). (A) LVEF was measured by echocardiography 3 weeks after treatment. (B) Treatment effects (changes in LVEF at 3 weeks relative to baseline) were calculated for each group. (C and D) Representative Masson's trichrome staining of myocardial section 3 weeks after treatments. Scale bar: $1 \mathrm{~mm}$. (E-G) Quantitative analysis of infarct size, infarct wall thickness, and viable tissue from Masson's trichrome-stained images. $n=6$ animals per group. ${ }^{*} P<$ $0.05,{ }^{* *} P<0.01,{ }^{* * *} P<0.001$. Oneway ANOVA with Bonferroni post hoc correction. All values are mean \pm SD.

\section{Discussion}

Our results suggest that FEXO has impaired therapeutic potency in an animal model of MI and is incapable of promoting angiomyogenesis in vitro and in vivo. miRNA and a series of gain- and loss-offunction experiments revealed that the impairment of regenerative activities of FEXO is related to miR-21-5p dysregulation (Figure 8J).

Over the past decade, cell therapy has emerged as a promising therapeutic option for cardiac regeneration and protection. As a major paracrine component, exosomes have been revealed to play a vital role in tissue repair by packaging and delivering RNAs and proteins. It has been reported that injured mouse hearts treated with cardiac stem cell-secreted exosomes exhibited regenerative and functional improvements produced by the stem cells themselves $(21,36)$. Exosomes derived from mouse embryonic stem cells promote neovascularization and cardiomyocyte survival and reduce fibrosis after MI, consistent with the resurgence of the car- diac proliferative response produced by embryonic stem cells (42). However, most of these exosome products are derived from young and healthy donors. Because most patients with cardiac diseases, especially heart failure, suffer from systemic inflammation and are elderly, it is possible that heart failure modifies exosome contents and further compromises their reparative properties. In this study, FEXO lost proangiogenic drive of HUVEC tube formations and failed to promote cardiomyocyte proliferation in injured mouse hearts. This line of evidence suggests a dysfunction of FEXO in ischemic tissue repair. Our finding can partly explain the compromised therapeutic effects in clinical trials using autologous stem cells. The advanced cardiomyopathy condition modifies exosome contents and renders the stem cells ineffective in therapeutic repair.

The mechanisms that drive exosome-mediated repair rely on the transfer of specific donor cell miRNAs and proteins to recipient cells (43). In the present study, we performed miRNA array 
A
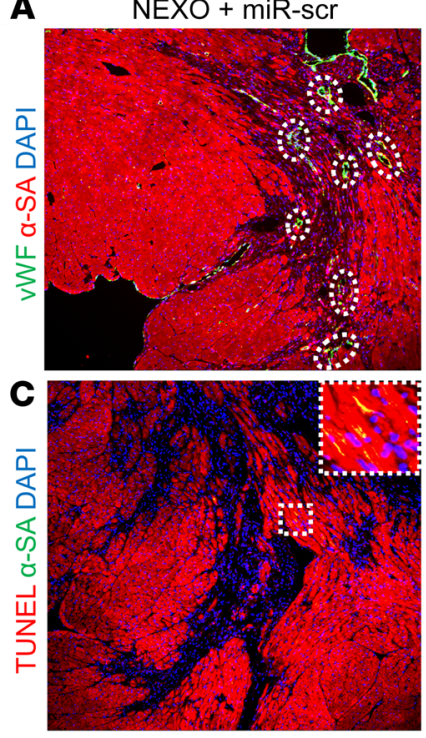

$\mathbf{E}$
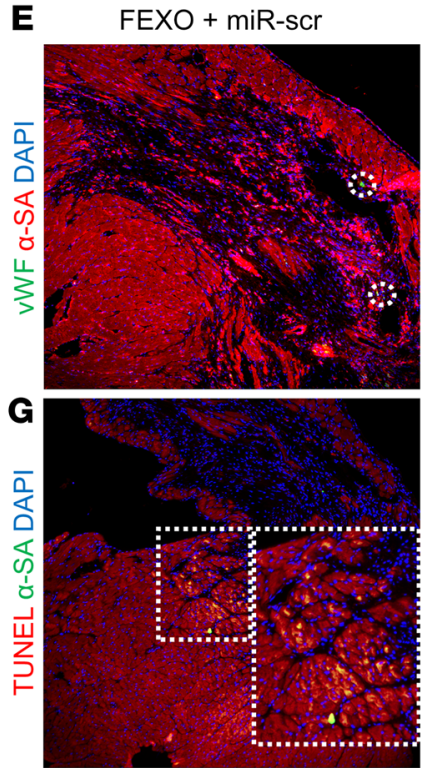

$\mathrm{NEXO}+$ anti-miR-21
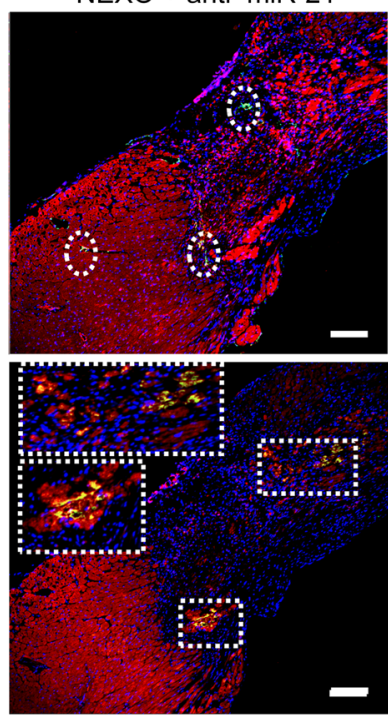

$\mathrm{FEXO}+\mathrm{miR}-21$
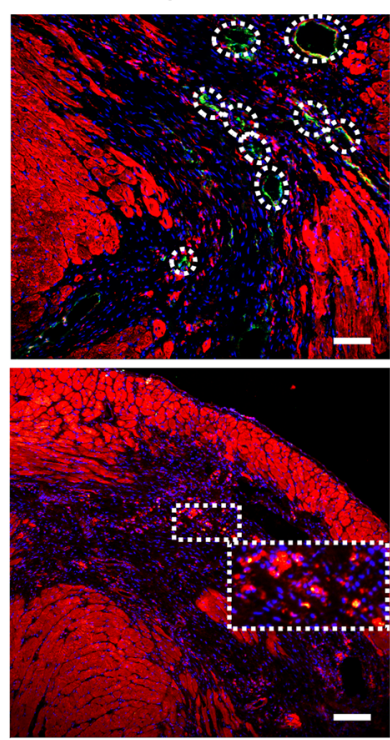

B
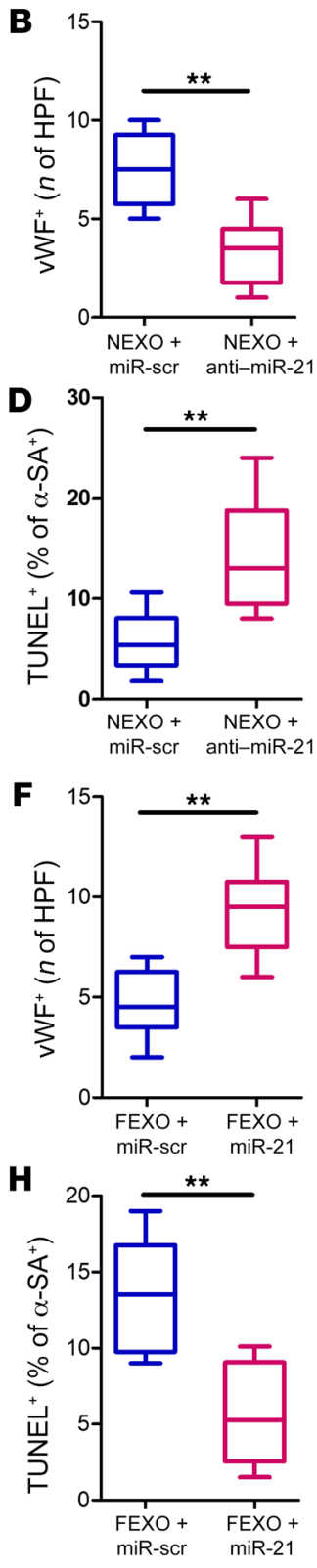

Figure 7. Manipulation of miR21 in exosomes modulates their proangiogenic and antiapoptotic activities in vivo. (A) Representative images of post-MI heart sections stained with vWF (green), $\alpha$-SA (red), and DAPI (blue). White circles indicate capillaries in the periinfarct zone. Scale bar: $100 \mu \mathrm{m}$. (B) Quantitation of capillary density (vWF+). (C) Heart sections stained with TUNEL (green), $\alpha$-SA (red), and DAPI (blue). White squares indicate apoptotic cardiomyocytes in the peri-infarct zone. Scale bar: $50 \mu \mathrm{m}$. (D) Quantitation of cardiomyocyte apoptosis (TUNEL+/a-SA+).

(E) Representative images of post-MI heart sections stained with vWF (green), $\alpha-S A$ (red), and DAPI (blue). White circles indicate capillaries in the peri-infarct zone. Scale bar: $50 \mu \mathrm{m}$. (F) Quantitation of capillary density $\left(\mathrm{VWF}^{+}\right)$. (G) Heart sections stained with TUNEL (green), $\alpha$-SA (red), and DAPI (blue). White squares indicate apoptotic cardiomyocytes in the peri-infarct zone. Scale bar: $50 \mu \mathrm{m}$. (H) Quantitation of cardiomyocyte apoptosis (TUNEL $\left.{ }^{+} / \alpha-S A^{+}\right)$. (B, D, F, and $\left.\mathbf{H}\right) n=6$ animals for each group and 3 heart sections for each animal. ${ }^{* *} P<0.01$. Two-tailed $t$ test. All values are mean $\pm \mathrm{SD}$. FEXO + miR-scr, exosomes derived from the cardiac cells of patients with heart failure transfected with scrambled miR oligo. FEXO + miR-21, exosomes derived from the cardiac cells of patients with heart failure transfected with miR-21-5p oligo. NEXO + miRscr, exosomes derived from the cardiac cells of the normal hearts transfected with scrambled miR oligo. NEXO + anti-miR-21, exosomes derived from the cardiac cells of the normal hearts transfected with anti-miR-21-5p oligo. analysis to compare FEXO with NEXO. We found that exosomal miR-21-5p, secreted from healthy cardiac cells, can be delivered to recipient cardiac cells to regulate apoptosis and angiogenesis and improve heart function in a mouse MI model. The silencing of miR-21-5p in NEXO eliminated these benefits, and the restoration of miR-21-5p in FEXO rescued their therapeutic potency, further supporting the essential role of miR-21-5p in exosome-mediated heart repair. Although our studies focused on miR-21-5p, there are several other miRs dysregulated in FEXO, such as miR-146a, let-7b, miR-125a, and miR-23. It has been reported that exosomal miR-146a leads to beneficial effects in post-MI hearts by targeting Irak-1 and Traf6, both associated with the toll-like receptor signaling pathway (36). It has also been shown that miR-23 can promote angiogenesis in cardiac endothelial cells by activating proangiogenic signaling through the inhibition of Spry2 and Sema6A (44, 45). Our results indicate that miR-146a inhibited apoptosis in cardiomyocytes but promoted tube formation in endothelial cells, which is consistent with previous studies, while neither let-7b-5p nor miR-125a-5p showed beneficial effects on cardiomyocyte survival or endothelial tube formation.

Although miR-21 expression has been associated with various disorders $(39,46-48)$, its precise functional role in cardiac diseases is still controversial. Thomas et al. reported that miR-21 expression is highly upregulated by cardiac stress, leading to activation of ERK/MAP kinase signaling through inhibition of Spry1, which in turn promoted cardiac fibrosis and heart dysfunction (39). miR-21 also reportedly induced cardiac fibrosis by inhibiting PTEN expression in CFs at the infarct zone after myocardial ischemia-reperfusion (49). However, Patrick et al. reported that miR21 was not essential for cardiac fibrosis, as miR-21-null mice still display cardiac hypertrophy, fibrosis, and the loss of cardiac contractility (48). In the present study, miR-21 did not affect the survival of CFs (Supplemental Figure 11G). Moreover, miR-21 regulated the PTEN/Akt signaling pathway in cardiomyocytes and endothe- 
A
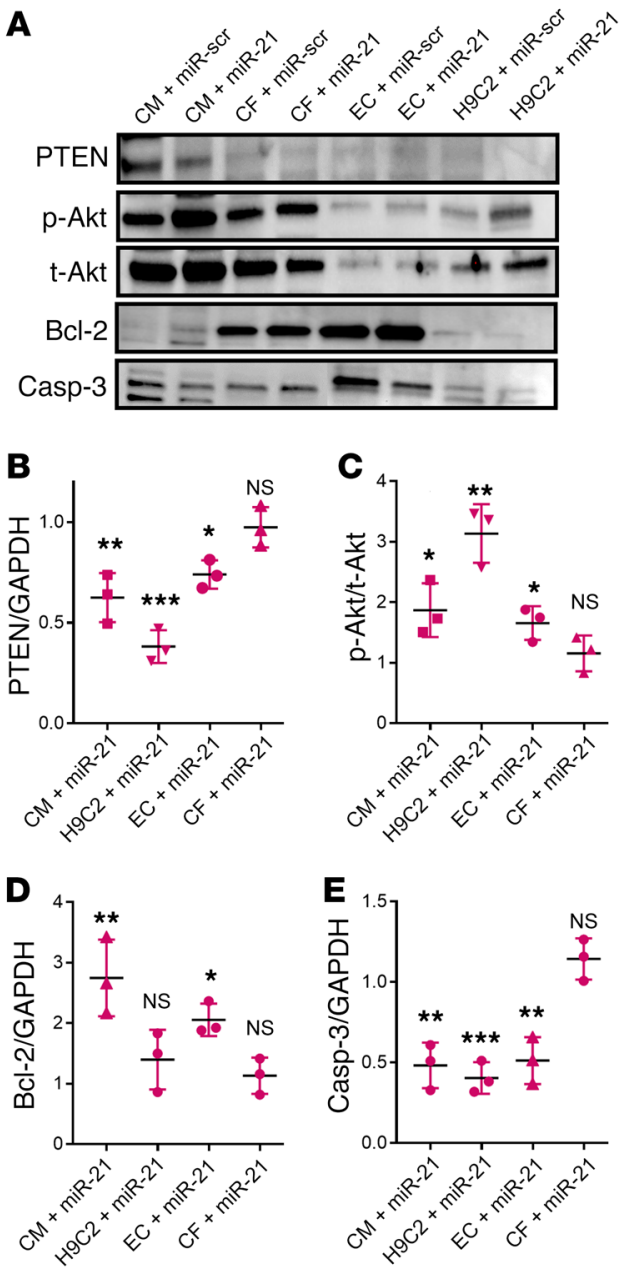

Figure 8. miR-21-5p targets the PTEN pathway in post-MI pathology. (A) Representative Western blot images showing the expression of various PTEN/Akt pathway components. (B-E) Quantitation of the levels of PTEN, p-Akt, t-Akt, Bcl-2, and caspase-3 $(n=3)$. (F) Representative Western blot images showing the expression of PCNA (proliferation marker), VEGF, and PDCD4 (miR-21 target). ( $(\mathbf{G}$ and $\mathbf{H})$ Quantitation of the levels of PCNA, VEGF, and PDCD4 $(n=3)$. (B-I) All miR-21 groups were normalized to the relevant scrambled controls. ${ }^{*} P<$ 0.05 compared to scrambled control. ${ }^{* *} P$ $<0.01$ compared to scrambled control. ${ }^{* * *} P<0.001$ compared to scrambled control. NS, no significance compared to scrambled control. Two-tailed $t$ test. All values are mean \pm SD. (J) Schematic showing the working model of our study. $\mathrm{CM} / \mathrm{HgC2} / \mathrm{EC} / \mathrm{CF}+$ miR-scr, $\mathrm{HCs} / \mathrm{HgC2}$ cells/HUVECs/human cardiac fibroblasts transfected with scrambled miR. CM/ $\mathrm{H} 9 \mathrm{C} 2 / \mathrm{EC} / \mathrm{CF}+\mathrm{miR}-21, \mathrm{HCs} / \mathrm{HgC2}$ cells/ HUVECs/human cardiac fibroblasts transfected with miR-21-5p mimic.

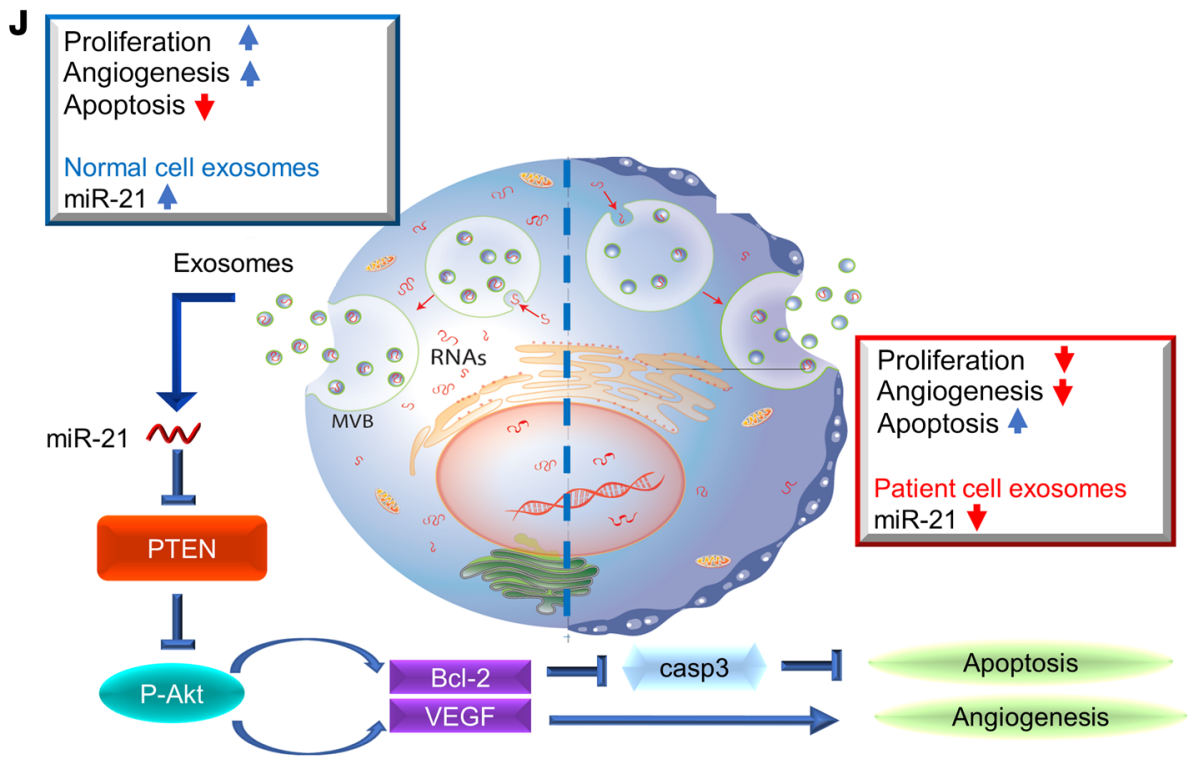

lial cells instead of fibroblasts (Figure 8), indicating that miR-21 does not promote cardiac fibrosis, as reported in previous studies.

In line with our findings, it was reported that exosomal miR-21 secreted by induced pluripotent stem cells promoted angiogenesis in transfected bronchial epithelial cells (50). Kristin et al. recently reported that miR-21a-5p is the most abundant miR in murine mesenchymal stem cell (MSC) exosomes and that it contributes to myocardial salvage via synergistic antiapoptotic activity (51). miR21-5p plays a crucial role in the MSC exosome-mediated effects on cardiac contractility through PI3K signaling (52). One unique aspect of our study is the cell source. Although therapeutic MSCs can be readily derived from bone marrow and adipose tissues, these 
cells do not naturally reside in the heart. Our study is more physiologically relevant. We focused on intrinsic cardiac cells in failing or healthy hearts and demonstrated that heart failure pathology leads to a severe dysregulation of miR-21-5p in intrinsic cardiac cells, which further leads to the impaired regenerative function of the intrinsic heart exosomes. Therefore, our findings demonstrate that miR-21 not only plays a key role in therapeutic interventions (e.g., in the case of MSCs) but also represents a crucial component in the naturally occurring repair process after heart injury.

The regulation of PTEN by miR-21 was reported in carcinoma (53) and cardiovascular cells (49). Consistent with this, we found that upregulation of miR-21-5p in exosomes augmented phosphorylation of Akt via the inhibition of PTEN in the recipient cells. These effects were eliminated by the knockdown of miR-21-5p, indicating a possible molecular mechanism for the positive role of miR-21-5p in post-MI repair. Based on the flow cytometry data, cardiac stromal cells/fibroblasts might be the main source of exosomes with altered miR-21. Thomas et al. (39) revealed strong miR21 expression primarily in CFs but was low in cardiomyocytes. This is consistent with our findings. It is quite possible that CFs released exosomal miR-21 to support survival of neighboring cardiomyocytes and endothelial cells under physiological conditions.

These findings have important clinical implications. Unlike cell-based therapy products, exosomes can offer an off-the-shelf and universal therapeutic option. The cryopreservation of exosomes normally does not require the sophisticated steps associated with cellular products. In addition, injection of allogeneic or even xenogeneic exosomes is well tolerated by the host immune system $(54,55)$. Our findings suggest that FEXO carries marked altered miR signatures compared with those from healthy heart cells and are functionally deficient in their ischemic myocardial repair capabilities. This may explain the reason why patients' autologous cell therapies only show modest therapeutic effects in clinical trials. More importantly, our data showed that the dysfunction of FEXO can be rescued by modulation of a specific miR in the exosomes. Our study provides mechanistic insights into the therapeutic potential of exosomes and strategies to rescue patients' defective exosomes through modulation of specific miR cargos. This discovery paves the ground for future translational efforts in fabricating "designer" and "super" exosomes for enhanced therapeutic applications.

There are some limitations in our study. First, HUVECs are not a good representation of cardiac endothelial cells. Heart primary endothelial cells are a more preferable source for in vitro studies. Second, the iPS-derived cardiomyocytes and the adult HC cell line we employed in our study do not completely resemble primary adult HCs. A third limitation of our study is that we only showed the effects of differential miR-21 expressions at the protein level but did not design an experiment (such as luciferase reporter assays or RT-PCR) to prove that miR-21-5p directly targets PTEN and PDCD4. However, such target validation results have been confirmed in the literature $(53,56)$. In addition, the CD1 mouse has been a tangible resource for biomedical research, including cardiovascular diseases. Nevertheless, we admit that the mouse MI model we employed has some limitations. The CD1 mice we used were relatively young. Therefore, their endogenous reparative ability does not resemble that of aged humans whose innate repair systems may be compromised, especially those suffering long-term heart failure.

\section{Methods}

Cell culture and exosome isolation. Diseased heart tissues were received from patients who underwent heart transplantation at UNC Hospital after heart failure. Healthy heart tissues were received from normal, nondamaged, human donor hearts. Cardiac cells were separated and cultured as previously described (6). In brief, myocardial tissues harvested from donors' hearts were separated and washed with PBS, cut into smaller 2- $\mathrm{mm}^{3}$ fragments, and partially enzymatically digested at $37^{\circ} \mathrm{C}$ with collagenase type IV (C1889, Sigma-Aldrich). The tissue samples were plated onto fibronectin-coated Petri dishes in Iscove's modified Dulbecco's medium (IMDM, Gibco) infused with 20\% fetal bovine serum (FBS; Corning). After 3 to 5 days, cardiac cells began to grow from the explants. Exosomes were isolated from the conditioned medium of these cardiac cells. Cardiac cells from passages 1 through 3 were cultured to $80 \%$ confluency. The medium was then switched to serum-free IMDM and conditioned for 14 days. Then, exosomes were isolated from the conditioned medium by ultrafiltration, as previously described $(54,55)$. In brief, conditioned medium was filtered through $0.22-\mu \mathrm{m}$ Steriflip filters to remove cellular debris and large vesicles. The filtrate was then added to Amicon Ultra-15 $100 \mathrm{kDa}$ filters (SCGP00525, Millipore) to centrifuge at 5,000 $\times g$ for 5 minutes. The flow-through was discarded. The concentrated exosomes were collected and washed with PBS 3 times and stored at $-80^{\circ} \mathrm{C}$. Labeling was performed using 10- $\mu$ M DiI (V22889, Thermo Fisher Scientific).

Flow cytometry. Flow cytometry was performed to examine the antigenic phenotypes of cardiac cells. Cells were incubated with antibodies against CD90 (555595, BD), CD105 (ab11414, Abcam), CD31 (555445, BD), CD 34 (ab81289, Abcam), CD 45 (555482, BD), and c-kit $(550412, \mathrm{BD})$ for 60 minutes at $4^{\circ} \mathrm{C}$. Both unstained and isotype controls $(555748,559320$, BD) were used as negative controls. Flow cytometry was conducted with a CytoFlex Flow Cytometer (Beckman Coulter) and data were analyzed with FCS Express software (De Novo).

Exosome characterization. Nanoparticle tracking analysis (NanoSight NS300, Malvern) was used to measure the concentration and size of exosomes. Each sample was imaged 5 times for 60 seconds and analyzed. Transmission electron microscopy was used to assess the morphology of exosomes. In preparation for transmission electron microscopy, exosomes were fixed with $4 \%$ paraformaldehyde and $1 \%$ glutaraldehyde and were stored at room temperature. Microscopy was conducted with a transmission electron microscope (JEM-2000FX, JEOL).

Immunocytochemistry. NRCMs and CFs were isolated and cultured as previously described (57). The H9C2 cell line was purchased from Sigma-Aldrich (88092904, Sigma-Aldrich). Adult HCs were purchased from Celprogen and cultured on $\mathrm{HC}$ cell culture extracellular matrix (36044-15, Celprogen). iCMs were kindly provided by Dr. Freytes (UNC/NC State Joint Department of Biomedical Engineering, Chapel Hill/Raleigh). After 3 days of cultivation, the culture medium was supplemented with $7 \times 10^{8}$ exosomes or PBS for an additional 24 hours. They were fixed with $4 \%$ paraformaldehyde and blocked with Protein Block Solution (DAKO) with $1 \%$ saponin (Sigma-Aldrich). Subsequently, the cells were stained with anti- $\alpha$-sarcomeric actin ( $\alpha$-SA, a7811, Sigma-Aldrich), anti-Ki-67 (ab15580, Abcam), anti- $\alpha$-smooth muscle actin (ab5694, Abcam), or anti-vWF (ab6994, Abcam) antibodies. Flour 488 or Texas-Red conjugated secondary antibodies (ab150117, 
ab6787, ab6719, ab150077, Abcam) were used for detection. Sections treated TUNEL (12156792910, Roche) were incubated for 30 minutes after the secondary antibody incubation. Cell nuclei were counterstained with 4',6-diamidino-2-phenylindole (DAPI). Images were taken using a fluorescent microscope (Olympus IX81; Olympus).

Angiogenesis assay. HUVECs were co-incubated with $1.5 \times 10^{8}$ exosomes for 24 hours, then plated on growth factor-deprived Matrigel (356230, Corning) to evaluate angiogenesis (58). Eight hours later, tube formation was examined with a white light microscope and analyzed with NIH Image J software.

miR-21-5p transfection. miR-21-5p oligo, anti-miR-21-5p, or scrambled miR oligo transfection was performed according to the XMIR exosome RNA packaging protocol (XMIR-21, AXMIR-21, XMIR-POS, SBI System Biosciences) with slight modifications. Briefly, cardiac cells were cultured to $70 \%$ confluency. miR-21-5p, anti-miR-21-5p, or scrambled miR oligo were mixed with a transfection reagent, then added to the cell culture at a final concentration of $20 \mathrm{nM}$. Cells were returned to the incubator for exosome production for 24 hours. Culture media was collected, and exosomes were isolated as previously described. Transfection efficiency was determined by performing RT-PCR on exosomes derived from the transfected cells. Hs_miR21_2 miScript Primer Assay (MS00009079, Qiagen) was performed according to the manufacturer's protocol. RT-PCR was performed using Roche Light Cycler 480 Instrument II (Roche) and relative exosome abundance levels were calculated using the delta-delta Ct method. miR-16 (MS00031493, Qiagen) was used as a reference control.

Animal studies. The acute MI model was created as previously described (1-4). Sixty 6-week-old female CD-1 mice (Charles River Labs) underwent left thoracotomy under general anesthesia. The left anterior descending coronary artery was permanently ligated. Subsequently, intramyocardial injections were performed at 4 sites in the peri-infarct zone. All mice were randomly assigned to the following groups: (a) NEXO, intramyocardial injection of $50-\mu \mathrm{l}$ PBS containing $30 \times 10^{9} \mathrm{NEXO}$; (b) FEXO, injection of $50-\mu \mathrm{l}$ PBS containing $30 \times 10^{9} \mathrm{FEXO}$; (c) control, injection of 50- $\mu \mathrm{l} \mathrm{PBS}$; and (d) sham, mice with sham surgery underwent the same procedures except for the permanent ligation.

To validate the role of miR-21-5p in exosome-mediated heart repair, we created the same MI models in mice. miR-21-5p-deficient exosomes were engineered by transfecting healthy cardiac cells with anti-miR-21-5p oligo (NEXO + anti-miR-21). We also produced miR21-rescued exosomes by transfecting heart failure cardiac cells with miR-21 RNA oligo (FEXO + miR-21) to harvest exosomes with miR-21$5 \mathrm{p}$ overexpression. Scrambled miR oligo was used as a control (NEXO/ FEXO + miR-scr): (a) NEXO + anti-miR-21, injection of $50-\mu l$ PBS containing $30 \times 10^{9} \mathrm{NEXO}+$ anti-miR-21; (b) NEXO + miR-scr, injection of 50- $\mu$ PBS containing $30 \times 10^{9} \mathrm{NEXO}+$ miR-scr; (c) FEXO + miR-21, injection of 50- $\mu$ PBS containing $30 \times 10^{9} \mathrm{FEXO}+$ miR-21; (d) FEXO + miR-scr, injection of 50- $\mu$ l PBS containing $30 \times 10^{9} \mathrm{FEXO}+$ miR-scr.

Heart function assessment. Cardiac function was measured by blinded echocardiography analysis using a Philips Cx-70 Ultrasound System with an L15-7io high-frequency probe. Each measurement was performed 3 times. Long-axis views were measured at the greatest left ventricular diameter. Left ventricular ejection fraction was measured from views taken through the infarcted area.

Heart morphometry. Animals were sacrificed 3 weeks after treatments. Six cryosections from each heart, collected at $400-\mu \mathrm{m}$ intervals, were stained with Masson's trichrome. Infarct size, infarct circumference, infarct wall thickness, and viable tissue in the risk area were measured with NIH Image J software, as previously described (1-4).

Antibody array and Western blot analysis. Adult HCs (CM, 3604415, Celprogen), iCMs, H9C2 cells (88092904, Sigma-Aldrich), human CFs (HCF, 306-05A, Sigma-Aldrich), and HUVECs (PCS-100-010, ATCC) were transfected with miR-21-5p mimic or scrambled control (miScript miRNA Mimic, 219600, Qiagen), according to the manufacturer's protocol with slight modifications. Briefly, cells were cultured to $70 \%$ confluency. miR-21-5p mimic or scrambled control was mixed with HiPerFect Transfection Reagent and culture medium without serum, at a final concentration of $5 \mathrm{nM}$. Cells were returned to the incubator for 48 hours. Cells were collected, lysed by RIPA Lysis Buffer (89900, Thermo Fisher Scientific), and centrifuged at 14,000 $\times g$ for 15 minutes to pellet the cell debris. The protein concentration was quantified with a BCA Protein Assay (23227, Thermo Fisher Scientific).

Protein from transfected iCM, H9C2, HCF, and HUVEC cells was used on the signaling pathway phosphorylation array (AAH-AKT-1-2, RayBiotech). The protein array assay was performed according to the manufacturer's protocol with slight modification. Briefly, the antibody membranes were blocked for 30 minutes at room temperature. The samples were then added and incubated overnight at $4^{\circ} \mathrm{C}$. The detection antibody cocktail was prepared, pipetted onto the membranes, and incubated overnight at $4^{\circ} \mathrm{C}$. Then, the samples were incubated with HRP anti-rabbit IgG and visualized with the ChemiDoc Touch Imaging System (ChemiDoc, Bio-Rad). Quantitative analysis was performed with Image J or Image Lab software.

Protein from transfected CM, H9C2, HCF, and HUVEC cells was used on Western blot analysis. The equivalent of $25 \mathrm{mg}$ of total protein per lane was loaded onto Mini-PROTEAN TGX Stain-Free Protein Gels (4568083, Bio-RAD) and transferred to Immun-Blot PVDF Membranes (1620177, Bio-RAD). Membranes were blocked with 5\% blocking buffer for 1 hour and incubated with primary antibodies against PTEN (ab31392, Abcam), p-akt (phospho T308) (ab38449, Abcam), t-akt (ab8805, Abcam), BCL-2 (ab59348, Abcam), caspase-3 (ab13847, Abcam), PDCD4 (ab51495, Abcam), PCNA (ab29, Abcam), or VEGF (PA5-16754, Thermo Fisher Scientific) overnight, at $4^{\circ} \mathrm{C}$ on a rocker. Subsequently, the samples were incubated with appropriate horseradish peroxidase-conjugated secondary antibodies at room temperature for 1 hour on a shaker. The blots were exposed with Clarity Western ECL Blotting Substrates (1705061, Bio-Rad) and visualized with the ChemiDoc Touch Imaging System (ChemiDoc, Bio-Rad). Quantitative analysis was performed with ImageJ or Image Lab software, and expressions were normalized to GAPDH.

Statistics. GraphPad Prism (GraphPad Software) was used for statistical analysis. Results were presented as mean \pm SD. All comparisons between 2 groups were performed with 2-tailed unpaired Student's $t$-test. One-way ANOVA analysis, with post hoc Bonferroni correction, was used to compare means among more than 2 groups. Differences were considered statistically significant when $P<0.05$.

Study approval. All animal procedures were approved by the Institutional Animal Care and Usage Committee (IACUC, North Carolina State University).

\section{Author contributions}

YL and KC designed the overall experiments. LQ, SH, SL, HZ, $\mathrm{HM}, \mathrm{KH}, \mathrm{AV}$, TS, ZL, JT, JC, TA, PUD, and QY performed the 
experiments and analyzed the data. LQ, JC, and $\mathrm{KC}$ wrote the article. All authors have approved the final article. All authors have provided the corresponding author with written permission to be named in the article.

\section{Acknowledgments}

This study was sponsored by the NIH (HL123920, HL137093, HL144002, and HL146153 to KC), National Natural Science Foundation of China (81570345 to YL, 81800267 to JT), and Henan Province Medicine and Technology Joint Project (201802002 to
JT). LQ is supported by China Scholarship Council. The funders had no role in study design, data collection, and interpretation, or the decision to submit the work for publication.

Address correspondence to: Yongjun Li, Professor of Cardiology, The Second Hospital of Hebei Medical University, 215 Heping Road, Shijiazhuang 050000, Hebei, China. Phone: 86.0311.66002126; Email: lyjbs2009@yeah.net. Or to: Li Qiao, The Second Hospital of Hebei Medical University, 215 Heping Road, Shijiazhuang 050000, Hebei, China. Phone: 86.0311.66006246; Email: qiaolixinnei@163.com.
1. Luo L, et al. Fabrication of synthetic mesenchymal stem cells for the treatment of acute myocardial infarction in mice. Circ Res. 2017;120(11):1768-1775.

2. Tang J, et al. Targeted repair of heart injury by stem cells fused with platelet nanovesicles. Nat Biomed Eng. 2018;2:17-26.

3. Tang J, et al. Heart repair using nanogel-encapsulated human cardiac stem cells in mice and pigs with myocardial infarction. ACS Nano. 2017;11(10):9738-9749.

4. Tang J, et al. Therapeutic microparticles functionalized with biomimetic cardiac stem cell membranes and secretome. Nat Commun. 2017;8:13724.

5. Kreke M, Smith RR, Marbán L, Marbán E. Cardiospheres and cardiosphere-derived cells as therapeutic agents following myocardial infarction. Expert Rev Cardiovasc Ther. 2012;10(9):1185-1194.

6. Makkar RR, et al. Intracoronary cardiospherederived cells for heart regeneration after myocardial infarction (CADUCEUS): a prospective, randomised phase 1 trial. Lancet. 2012;379(9819):895-904.

7. Chugh AR, et al. Administration of cardiac stem cells in patients with ischemic cardiomyopathy: the SCIPIO trial: surgical aspects and interim analysis of myocardial function and viability by magnetic resonance. Circulation.

2012;126(11 suppl 1):S54-S64.

8. Rhee JW, Wu JC. Cardiac cell cycle activation as a strategy to improve iPSC-derived cardiomyocyte therapy. Circ Res. 2018;122(1):14-16.

9. Qi CM, et al. Transplantation of magnetically labeled mesenchymal stem cells improves cardiac function in a swine myocardial infarction model. Chin Med J. 2008;121(6):544-550.

10. Bellamy V, et al. Long-term functional benefits of human embryonic stem cell-derived cardiac progenitors embedded into a fibrin scaffold. J Heart Lung Transplant. 2015;34(9):1198-1207.

11. Kim SW, Houge M, Brown M, Davis ME, Yoon YS. Cultured human bone marrow-derived CD31(+) cells are effective for cardiac and vascular repair through enhanced angiogenic, adhesion, and anti-inflammatory effects. J Am Coll Cardiol. 2014;64(16):1681-1694.

12. Joladarashi D, et al. Enhanced cardiac regenerative ability of stem cells after ischemia-reperfusion injury: role of human $\mathrm{CD} 34^{+}$cells deficient in microRNA-377. J Am Coll Cardiol. 2015;66(20):2214-2226.

13. Tang JN, et al. Concise review: is cardiac cell ther- apy dead? embarrassing trial outcomes and new directions for the future. Stem Cells Transl Med. 2018;7(4):354-359.

14. Fernández-Avilés F, et al. Global Overview of the Transnational Alliance for Regenerative Therapies in Cardiovascular Syndromes (TACTICS) Recommendations: a comprehensive series of challenges and priorities of cardiovascular regenerative medicine. Circ Res. 2018;122(2):199-201.

15. Chimenti I, et al. Relative roles of direct regeneration versus paracrine effects of human cardiosphere-derived cells transplanted into infarcted mice. Circ Res. 2010;106(5):971-980.

16. Malliaras K, et al. Safety and efficacy of allogeneic cell therapy in infarcted rats transplanted with mismatched cardiosphere-derived cells. Circulation. 2012;125(1):100-112.

17. Kittel A, Falus A, Buzás E. Microencapsulation technology by nature: Cell derived extracellular vesicles with therapeutic potential. Eur J Microbiol Immunol (Bp). 2013;3(2):91-96.

18. Raposo G, Stoorvogel W. Extracellular vesicles: exosomes, microvesicles, and friends. J Cell Biol. 2013;200(4):373-383.

19. Kalra H, Drummen GP, Mathivanan S. Focus on extracellular vesicles: introducing the next small big thing. Int J Mol Sci. 2016;17(2):170.

20. Barros FM, Carneiro F, Machado JC, Melo SA. Exosomes and immune response in cancer: friends or foes? Front Immunol. 2018;9:730.

21. Gallet R, et al. Exosomes secreted by cardiosphere-derived cells reduce scarring, attenuate adverse remodelling, and improve function in acute and chronic porcine myocardial infarction. Eur Heart J. 2017;38(3):201-211.

22. Mathiyalagan $P$, et al. Angiogenic mechanisms of human $\mathrm{CD} 34^{+}$stem cell exosomes in the repair of ischemic hindlimb. Circ Res. 2017;120(9):1466-1476.

23. Lai RC, Chen TS, Lim SK. Mesenchymal stem cell exosome: a novel stem cell-based therapy for cardiovascular disease. Regen Med. 2011;6(4):481-492.

24. Sahoo S, Losordo DW. Exosomes and cardiac repair after myocardial infarction. Circ Res. 2014;114(2):333-344.

25. Prathipati P, Nandi SS, Mishra PK. Stem cellderived exosomes, autophagy, extracellular matrix turnover, and miRNAs in cardiac regeneration during stem cell therapy. Stem Cell Rev. 2017;13(1):79-91.

26. Sluijter JP, van Rooij E. Exosomal microRNA clusters are important for the therapeutic effect of cardiac progenitor cells. Circ Res.
2015;116(2):219-221.

27. Wang Y, et al. Exosomes/microvesicles from induced pluripotent stem cells deliver cardioprotective miRNAs and prevent cardiomyocyte apoptosis in the ischemic myocardium. Int J Cardiol. 2015;192:61-69.

28. Yue Y, Garikipati VNS, Verma SK, Goukassian DA, Kishore R. Interleukin-10 deficiency impairs reparative properties of bone marrow-derived endothelial progenitor cell exosomes. Tissue Eng Part A. 2017;23(21-22):1241-1250.

29. Liu B, et al. Cardiac recovery via extended cellfree delivery of extracellular vesicles secreted by cardiomyocytes derived from induced pluripotent stem cells. Nat Biomed Eng. 2018;2(5):293-303.

30. Pang T, et al. Endothelial progenitor cells are influenced by serum of patients with systemic inflammatory response syndrome or multiple organ dysfunction. Eur Rev Med Pharmacol Sci. 2013;17(23):3169-3177.

31. Lin CP, et al. Endothelial progenitor cell dysfunction in cardiovascular diseases: role of reactive oxygen species and inflammation. Biomed Res Int. 2013;2013:845037.

32. Desouza CV, Hamel FG, Bidasee K, O'Connell K. Role of inflammation and insulin resistance in endothelial progenitor cell dysfunction. Diabetes. 2011;60(4):1286-1294.

33. Hong Y, Eleftheriou D, Klein NJ, Brogan PA. Impaired function of endothelial progenitor cells in children with primary systemic vasculitis. Arthritis Res Ther. 2015;17:292.

34. Théry C, Amigorena S, Raposo G, Clayton A. Isolation and characterization of exosomes from cell culture supernatants and biological fluids. Curr Protoc Cell Biol. 2006; Chapter 3:Unit 3.22.

35. Gnecchi M, et al. Paracrine action accounts for marked protection of ischemic heart by Akt-modified mesenchymal stem cells. Nat Med. 2005;11(4):367-368.

36. Ibrahim AG, Cheng K, Marbán E. Exosomes as critical agents of cardiac regeneration triggered by cell therapy. Stem Cell Reports. 2014;2(5):606-619.

37. Barile L, et al. Extracellular vesicles from human cardiac progenitor cells inhibit cardiomyocyte apoptosis and improve cardiac function after myocardial infarction. Cardiovasc Res. 2014;103(4):530-541.

38. Melnik BC. MiR-21: an environmental driver of malignant melanoma? J Transl Med. 2015;13:202.

39. Thum T, et al. MicroRNA-21 contributes to myocardial disease by stimulating MAP kinase signalling in fibroblasts. Nature. 2008;456(7224):980-984. 
40. Wang K, et al. Enhanced cardioprotection by human endometrium mesenchymal stem cells driven by exosomal microRNA-21. Stem Cells Transl Med. 2017;6(1):209-222.

41. Khan M, et al. Embryonic stem cell-derived exosomes promote endogenous repair mechanisms and enhance cardiac function following myocardial infarction. Circ Res. 2015;117(1):52-64.

42. Kervadec A, et al. Cardiovascular progenitorderived extracellular vesicles recapitulate the beneficial effects of their parent cells in the treatment of chronic heart failure. J Heart Lung Transplant. 2016;35(6):795-807.

43. Dhanabal M, et al. Recombinant semaphorin 6A-1 ectodomain inhibits in vivo growth factor and tumor cell line-induced angiogenesis. Cancer Biol Ther. 2005;4(6):659-668.

44. Impagnatiello MA, Weitzer S, Gannon G, Compagni A, Cotten M, Christofori G. Mammalian sprouty- 1 and -2 are membraneanchored phosphoprotein inhibitors of growth factor signaling in endothelial cells. J Cell Biol. 2001;152(5):1087-1098.

45. Selcuklu SD, Donoghue MT, Spillane C. miR-21 as a key regulator of oncogenic processes. Biochem Soc Trans. 2009;37(pt 4):918-925.
46. Thum T, et al. MicroRNA-21 contributes to myocardial disease by stimulating MAP kinase signalling in fibroblasts. Nature. 2008;456(7224):980-984.

47. Bang C, et al. Cardiac fibroblast-derived microRNA passenger strand-enriched exosomes mediate cardiomyocyte hypertrophy. J Clin Invest. 2014;124(5):2136-2146.

48. Patrick DM, et al. Stress-dependent cardiac remodeling occurs in the absence of microRNA-21 in mice. J Clin Invest. 2010;120(11):3912-3916.

49. Roy S, et al. MicroRNA expression in response to murine myocardial infarction: $\mathrm{miR}-21 \mathrm{reg}-$ ulates fibroblast metalloprotease-2 via phosphatase and tensin homologue. Cardiovasc Res. 2009;82(1):21-29.

50. Xu Y, et al. Exosomal miR-21 derived from arsenite-transformed human bronchial epithelial cells promotes cell proliferation associated with arsenite carcinogenesis. Arch Toxicol. 2015;89(7):1071-1082.

51. Luther KM, et al. Exosomal miR-21a-5p mediates cardioprotection by mesenchymal stem cells. JMol Cell Cardiol. 2018;119:125-137.

52. Mayourian J, et al. Exosomal microRNA-21-5p mediates mesenchymal stem cell paracrine effects on human cardiac tissue contractility. Circ Res. 2018;122(7):933-944.

53. Meng F, Henson R, Wehbe-Janek H, Ghoshal K, Jacob ST, Patel T. MicroRNA-21 regulates expression of the PTEN tumor suppressor gene in human hepatocellular cancer. Gastroenterology. 2007;133(2):647-658.

54 . Vandergriff A, et al. Targeting regenerative exosomes to myocardial infarction using cardiac homing peptide. Theranostics. 2018;8(7):1869-1878.

55 . Vandergriff AC, et al. Intravenous cardiac stem cell-derived exosomes ameliorate cardiac dysfunction in doxorubicin induced dilated cardiomyopathy. Stem Cells Int. 2015;2015:960926.

56. Asangani IA, et al. MicroRNA-21 (miR-21) post-transcriptionally downregulates tumor suppressor Pdcd4 and stimulates invasion, intravasation and metastasis in colorectal cancer. Oncogene. 2008;27(15):2128-2136.

57. Vandergriff AC, Hensley MT, Cheng K. Isolation and cryopreservation of neonatal rat cardiomyocytes. J Vis Exp. 2015;(98):e52726.

58. Manoussaki D, Lubkin SR, Vernon RB, Murray JD. A mechanical model for the formation of vascular networks in vitro. Acta Biotheor. 1996;44(3-4):271-282. 